\title{
Linear pulse structure and signalling in a film flow on an inclined plane
}

\author{
By LEONID BREVDO ${ }^{1} \dagger$, PATRICE LAURE \\ FREDERIC DIAS ${ }^{2}$ AND THOMAS J. BRIDGES \\ ${ }^{1}$ Mathematisches Institut A, Universität Stuttgart, Pfaffenwaldring 57, \\ D-70569 Stuttgart, Germany \\ ${ }^{2}$ Institut Non-Linéaire de Nice (UMR CNRS 6618), Université de Nice, Sophia Antipolis, \\ 06560 Valbonne, France \\ ${ }^{3}$ Department of Mathematics and Statistics, University of Surrey, Guildford, Surrey, \\ GU2 5XH England, UK
}

(Received 21 September 1998 and in revised form 21 April 1999)

The film flow down an inclined plane has several features that make it an interesting prototype for studying transition in a shear flow: the basic parallel state is an exact explicit solution of the Navier-Stokes equations; the experimentally observed transition of this flow shows many properties in common with boundary-layer transition; and it has a free surface, leading to more than one class of modes. In this paper, unstable wavepackets - associated with the full Navier-Stokes equations with viscous free-surface boundary conditions-are analysed by using the formalism of absolute and convective instabilities based on the exact Briggs collision criterion for multiple $k$-roots of $D(k, \omega)=0$, where $k$ is a wavenumber, $\omega$ is a frequency and $D(k, \omega)$ is the dispersion relation function.

The main results of this paper are threefold. First, we work with the full NavierStokes equations with viscous free-surface boundary conditions, rather than a model partial differential equation, and, guided by experiments, explore a large region of the parameter space to see if absolute instability-as predicted by some model equations-is possible. Secondly, our numerical results find only convective instability, in complete agreement with experiments. Thirdly, we find a curious saddle-point bifurcation which affects dramatically the interpretation of the convective instability. This is the first finding of this type of bifurcation in a fluids problem and it may have implications for the analysis of wavepackets in other flows, in particular for three-dimensional instabilities. The numerical results of the wavepacket analysis compare well with the available experimental data, confirming the importance of convective instability for this problem.

The numerical results on the position of a dominant saddle point obtained by using the exact collision criterion are also compared to the results based on a steepest-descent method coupled with a continuation procedure for tracking convective instability that until now was considered as reliable. While for two-dimensional instabilities a numerical implementation of the collision criterion is readily available, the only existing numerical procedure for studying three-dimensional wavepackets is based on the tracking technique. For the present flow, the comparison shows a failure of the tracking treatment to recover a subinterval of the interval of unstable ray velocities $V$ whose length constitutes $29 \%$ of the length of the entire unstable interval of $V$. The failure occurs due to a bifurcation of the saddle point, where $V$

$\dagger$ Present address: Ecole Supérieure de Mécanique de Marseille and IRPHE (UMR CNRS 6594), Université de la Méditerranée, IMT-Technopôle de Château-Gombert, F 13451 Marseille cedex 20, France. 
is a bifurcation parameter. We argue that this bifurcation of unstable ray velocities should be observable in experiments because of the abrupt increase by a factor of about 5.3 of the wavelength across the wavepacket associated with the appearance of the bifurcating branch. Further implications for experiments including the effect on spatial amplification rate are also discussed.

\section{Introduction}

Waves at the surface of a fluid, particularly water waves, have been a fascinating area of enquiry for as long as there are records. Therefore it is not surprising that some of the oldest problems in fluid mechanics involve waves on the surface of a fluid, and interest in such problems seems to be attracting more attention with time. Among such problems are two classical ones: the water-wave problem, directly related to the waves that one observes on the ocean, and the thin-film problem, that can be directly related to waves that one observes on a sloping roadway in the rain, and to industrial applications in chemical engineering, for example. However the character of these two problems is quite different: the analysis of the first one is based on inviscid and irrotational flow theory - potential theory - while the analysis of the second one is based on viscous flow theory - the Navier-Stokes equations. References to the waterwave problem date as far back as 1847 (Stokes 1847), while references to the viscous flow of a thin film go back to 1916 (Nusselt 1916).

Both of the above wave problems deal initially with perturbations about a basic state: a uniform state of rest or uniform current for the water-wave problem and a steady gravity-driven parabolic velocity profile for the thin film flow. In the water-wave problem, the linearization about the basic state admits plane wave solutions satisfying a dispersion relation which is known analytically and is real for real values of the wavenumber and frequency. In the thin-film-wave problem, the linearization about the parabolic velocity profile leads to the Orr-Sommerfeld equation with boundary conditions coupled to the free-surface displacement, and the associated dispersion relation is in general complex valued, and except for special limiting cases, can only be obtained numerically.

In both cases the full nonlinear problem is difficult to tackle and has defied any comprehensive treatment, although the nonlinear water-wave problem has been studied in more detail than the nonlinear viscous thin-film problem. The greatest success in both problems has been achieved by analysing model equations, which are valid for limited regions of parameter space. Examples of model equations that have been proposed for water waves are the Korteweg-de Vries equation, the nonlinear Schrödinger equation, the Boussinesq systems, the Davey-Stewartson equation and the Zakharov equation. Examples of model equations for viscous thinfilm flow are the Benney equation (Benney 1966), a kinematic-wave equation (Mei 1966), the Roskes equation (Roskes 1970), a complex Ginzburg-Landau model (Lin 1974), and the Kuromoto-Sivashinsky equation (Chang \& Demekhin 1995). A model equation governing the nonlinear long-wave dynamics was derived by Lee \& Mei (1996) by applying the approximate momentum integral method of von Kármán. The most sophisticated model to date is the boundary-layer model proposed by Chang, Demekhin \& Kopelevich (1993) which includes variations in the transverse direction.

In the case of irrotational water waves as well as in the case of viscous thin-film flow, the question of stability is of primary importance. For water waves, the stability 
of weakly nonlinear Stokes' waves has been widely studied leading to the BenjaminFeir instability. It is interesting to note that the absolute or convective character of this instability has never been studied. For thin films the question of linear stability is already important for the basic state (cf. Kapitza \& Kapitza 1949) and has been an active area of research since the early work of Benjamin and Yih in the 1950s (Benjamin 1957, 1961; Yih 1955), based on approximate solutions of the OrrSommerfeld equation coupled with the free-surface boundary conditions. A stability analysis of the periodic states bifurcating from the neutral curve was first given by Lin (1974) who derived a complex Ginzburg-Landau model. Lin showed that there is an Eckhaus boundary within which the primary periodic states are stable to sideband perturbations. However, an analysis of the initial-value problem and a test for absolute or convective instability of the primary or secondary instability has not been given.

Experimental evidence and analysis of model equations show that the absolute and convective dichotomy is important for both the primary and the secondary instabilities observed in viscous film flow. Recent experiments of Liu \& Gollub (1993, 1994) and Liu, Paul \& Gollub (1993) confirmed the primary neutral stability curve of Benjamin and Yih, and several new observations were made as well. In Liu et al. (1993) strong evidence was presented for convective instability in all cases. In Liu \& Gollub (1993) unstable periodic waves were tracked until they developed a spatially chaotic structure. For the range of parameters studied, they also found that the secondary instability was strictly convective. They also note that secondary instabilities in thin-film flow have many features in common with instabilities in other shear flows such as boundary layers (see also the comments on this in Benjamin 1961). A remarkable new development in the experimental results of Liu, Schneider \& Gollub (1995) is the important role of three-dimensionality: they found synchronous three-dimensional instabilities, subharmonic instabilities and resonant triads; these three features are also prominently observed in transitional boundary layers.

Recent results on model equations for thin-film flow have been reviewed in Chang (1994) and Chang \& Demekhin (1996). Theoretical aspects of the classification of instabilities as absolute or convective for thin-film flow have been addressed in Joo \& Davis (1992). In that paper the role of absolute and convective instabilities associated with the primary instability of thin-film flow was studied using a model equation and they found predominantly convective instability; but they also found regions in parameter space where the instability is absolute. The linearized model equation used in the analysis of Lin (1974) is absolutely unstable for all parameter values in the unstable range. In Chang \& Demekhin (1996) the falling-film flow was shown to be absolutely stable for all Reynolds numbers below 500 by treating the full linearized Navier-Stokes equations.

In this paper we study the initial-value problem for the full Navier-Stokes equations linearized about the basic parabolic velocity profile for thin-film flow analytically, using the Fourier-Laplace transform to reduce the linearized Navier-Stokes equations to an inhomogeneous Orr-Sommerfeld equation coupled to inhomogeneous boundary conditions. The resulting boundary-value problem is treated numerically using a spectral (Chebyshev polynomials) method. Our main results are threefold. First, we give an absolute and convective instability classification of the full problem and describe properties of unstable wavepackets for a large and experimentally relevant range of the parameter space. Secondly, we show that absolute instability predicted by the model equations in Joo \& Davis (1992) and in Lin (1974) is anomalous and is not a property of the full equations-indeed, for all values of parameters studied here we find that the primary instability is convective, in agreement with all known 
experiments. Thirdly, we find that a straightforward application of the saddle-point technique even in an apparently accurate way to discriminate between absolute and convective instabilities fails due to an interesting bifurcation that takes place in the complex wavenumber plane.

The basic fact that the existence of an unstable saddle point is not sufficient for absolute instability has been recognized since the early days of the development of the theory of unstable wavepackets in the 1950s. In Briggs (1964), a mathematical example of an unstable saddle point making no contribution to the instability was given and a condition was derived, namely a collision criterion, which must be satisfied by a contributing saddle point. Brevdo (1988) has shown analytically that for the Eady model of a geophysical flow there exists a countable set of unstable saddle points and none of these points contributes to the instability. Recently, Lingwood (1997) has found numerically examples of such saddle points for a rotating boundary layer flow. Since an application of the Briggs (1964) collision criterion is numerically more expensive than a saddle-point treatment, procedures have been developed for applying the saddle-point technique without performing a direct collision check. One such procedure was proposed by Kupfer, Bers \& Ram (1987) for studying twodimensional instabilities. It is based on mapping the real $k$-axis into the complex $\omega$-plane under the transformations $\omega=\omega_{m}(k)$, where $\omega_{m}(k), m=1,2, \ldots$, are all the unstable branches of frequency, and analysing the saddle points of $\omega=\omega_{m}(k)$, $m=1,2, \ldots$, in the complex $\omega$-plane that are located between the image curves under these mappings and the real $\omega$-axis. While this technique is quite valuable for two-dimensional dispersion relations, it is not clear how to extend this procedure to three-dimensional instabilities.

A method for tracking the movement of saddle points which is applicable to both two-dimensional and three-dimensional instabilities and has heretofore been viewed as reliable is based on a continuation procedure. In the two-dimensional case such an application starts with the saddle point $k_{m}=\left(k_{m r}, 0\right)$ of the function $\omega(k)-V_{g} k$ on the real $k$-axis, where $\omega=\omega(k)$ is the unstable branch of frequency, and $V_{g}=\partial \omega_{r}\left(k_{m r}, 0\right) / \partial k_{r}$ is the group velocity of the unstable wavepacket, with $\omega_{i}\left(k_{m r}, 0\right)=$ maximum for real $k$. The subscripts $r$ and $i$ of $k$ and $\omega$ denote the real and imaginary parts, respectively, and $\omega_{r}$ and $\omega_{i}$ are considered as functions of two real variables $k_{r}$ and $k_{i}$. The saddle point $k_{m}$ makes the dominant contribution to the instability along the most unstable ray $x=x_{0}+V_{g} t$. The tracking (continuation) procedure follows the evolution of the saddle point starting with $k_{m}=\left(k_{m r}, 0\right)$, when the ray velocity $V$ varies continuously starting with $V_{g}$ (Simmons \& Hoskins 1979; Deissler 1987; Brevdo 1995). In the three-dimensional case the procedure is similar.

In this paper, we demonstrate the inconsistency of this tracking procedure. This observation is fundamental and could have wide implications in fluid mechanics where simple saddle-point criteria are applied, in particular for three-dimensional instabilities. In the three-dimensional case, the approach proposed by Kupfer et al. (1987) does not seem to be applicable, and at the present time there is no numerical algorithm for implementing the collision criterion for classifying three-dimensional instabilities derived by Brevdo (1991). In fact, at present the only numerical treatment of three-dimensional instabilities known to us is based on the continuation procedure. However, since the continuation procedure is shown here to be inconsistent, there exists practically no reliable numerical tool at the present time for distinguishing between absolute and convective three-dimensional instabilities. As far as we are aware, the inconsistency of the saddle-point treatment based on the continuation procedure is the first observation of this type of dispersion-relation singularity in a 


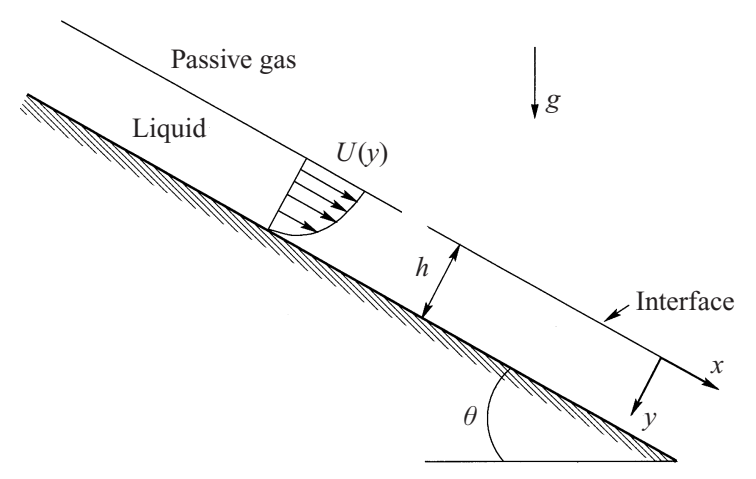

FIGURE 1. Geometric configuration and coordinate system.

fluid mechanics problem. It is central to the thin-film problem and we will argue that it should also be observable in experiments.

The paper is organized as follows. In $\S 2$ the model is described and the linear initialvalue problem is formulated. A formal solution of the problem is given in Appendix A. Procedures for determining the asymptotics of the solution based on the collision criterion and on the saddle-point approach are discussed in $\S 3$ and in Appendix B. Convectively unstable wavepackets are treated in $\S 4$, and the inconsistency of the saddle-point procedure outlined above is shown. In $\S 5$ computations of spatially amplifying waves are presented, and a comparison with experiments is made. The computed spatial growth rates are compared with the approximate results obtained by using the Gaster transformation. In the present case, the approximation given by the Gaster transformation is practically indistinguishable from the computed results in the entire range of unstable modes, for all Reynolds numbers considered. Finally, in $\S 6$ we summarize the findings in the paper, address further the physical implications of the results and the potential for observability in experiments, and discuss the implications of the saddle-point bifurcation discovered here for other calculations of saddle points, particularly for three-dimensional instabilities.

\section{Formulation}

We consider a two-dimensional film flow of homogeneous incompressible viscous fluid of viscosity $\mu$ and density $\rho$ down an inclined flat plate having an angle $\theta$ with the horizontal, see figure 1. The film thickness in the absence of disturbances is $h$, the gas above the film is assumed to be passive, the surface tension on the interface between the fluid and the gas is $T$. The basic unidirectional flow parallel to the plate is driven by the component $g \sin \theta$ of gravity along the plate. There is a basic hydrostatic pressure gradient perpendicular to the plate induced by the component $g \cos \theta$ of gravity normal to the plate. In the coordinate system $(O x y)$, with $x$ and $y$ being the coordinates parallel and perpendicular to the plate, respectively, and with the origin $O$ on the unperturbed interface, the non-dimensional basic state is given by

$$
U(y)=1-y^{2}, \quad P(y)=2 y \cot \theta, \quad 0 \leqslant y \leqslant 1,
$$

where $U(y)$ is the $x$-component of the velocity vector and $P(y)$ is the pressure. All lengths are made dimensionless with respect to $h$, all speeds with respect to $U_{0}=\left(\rho g h^{2} \sin \theta\right) / 2 \mu$, and the pressure is scaled by $\mu U_{0} / h$. 
The two-dimensional linear perturbation dynamics of the film flow is governed by the Navier-Stokes equations linearized around the basic state (2.1) that, in the absence of external sources and perturbations, read

$$
\begin{aligned}
R u_{t}+R\left[U(y) u_{x}+U^{\prime}(y) v\right] & =-p_{x}+\nabla^{2} u, \\
R v_{t}+R U(y) v_{x} & =-p_{y}+\nabla^{2} v, \\
u_{x}+v_{y} & =0, \\
(y, x, t) & \in(0,1) \times \mathbb{R} \times \mathbb{R}^{+} .
\end{aligned}
$$

Here, $u$ and $v$ are the $x$ - and $y$-components of the perturbation velocity, respectively, $p$ is the perturbation pressure, $R=U_{0} h \rho / \mu=\rho^{2} g h^{3} \sin \theta /\left(2 \mu^{2}\right)$ is the Reynolds number, $\nabla^{2}=\partial^{2} / \partial x^{2}+\partial^{2} / \partial y^{2}$, and the prime denotes $\mathrm{d} / \mathrm{d} y$. When no disturbances are imposed on the boundaries, (i) the interface $y=\eta(t, x)$ is composed of flow trajectories (the kinematic condition), (ii) the shear stress at the interface is zero, (iii) the normal stress at $y=\eta(t, x)$ is balanced by the surface tension times the curvature (two dynamic conditions), and (iv) the fluid velocity vanishes at the plate $y=1$ (the non-penetration and non-slip conditions). These conditions linearized around the basic state (2.1) are

$$
\begin{aligned}
\eta_{t}+U \eta_{x}-v=0 & \text { at } y=0, \\
u_{y}+v_{x}+U^{\prime \prime} \eta=0 & \text { at } y=0, \\
2 v_{y}-p-P^{\prime} \eta+2 W \eta_{x x}=0 & \text { at } y=0, \\
u=0, \quad v=0 & \text { at } y=1 .
\end{aligned}
$$

In (2.3c), $W=\Gamma /\left(g h^{2} \sin \theta\right)$ is the Weber number, with $\Gamma=T / \rho$.

We introduce the stream function $\Psi=\Psi(y, x, t)$. Elimination of pressure $p$ in $(2.2 a, b)$ and substitution of $u=\partial \Psi / \partial y, v=-\partial \Psi / \partial x$ result in the equation

$$
\left[R\left(\frac{\partial}{\partial t}+U \frac{\partial}{\partial x}\right) \nabla^{2}-R U^{\prime \prime} \frac{\partial}{\partial x}-\nabla^{4}\right] \Psi=0 .
$$

From (2.1) and (2.3b),

$$
\eta=\left(u_{y}+v_{x}\right) / 2, \quad \text { at } y=0 .
$$

We substitute (2.5) into (2.3a) and obtain, in terms of $\Psi$,

$$
\left(\frac{\partial}{\partial t}+\frac{\partial}{\partial x}\right)\left(\frac{\partial^{2} \Psi}{\partial y^{2}}-\frac{\partial^{2} \Psi}{\partial x^{2}}\right)+2 \frac{\partial \Psi}{\partial x}=0 \quad \text { at } y=0 .
$$

In order to eliminate $\eta$ in $(2.3 c)$ we differentiate it once with respect to $x$, eliminate $p_{x}$ in the resulting equation by using $p_{x}$ from $(2.2 a)$ evaluated at $y=0$, and make use of (2.5) to obtain

$$
\begin{aligned}
\frac{\partial^{3} \Psi}{\partial y^{3}}+\left(\cot \theta \frac{\partial}{\partial x}-W \frac{\partial^{3}}{\partial x^{3}}\right) & \left(\frac{\partial^{2} \Psi}{\partial y^{2}}-\frac{\partial^{2} \Psi}{\partial x^{2}}\right) \\
+ & 3 \frac{\partial^{3} \Psi}{\partial x^{2} \partial y}-R \frac{\partial}{\partial y}\left(\frac{\partial \Psi}{\partial t}+\frac{\partial \Psi}{\partial x}\right)=0 \quad \text { at } y=0
\end{aligned}
$$


The stream function formulation of $(2.3 d)$ is

$$
\Psi=0, \quad \frac{\partial \Psi}{\partial y}=0 \quad \text { at } y=1 .
$$

We are interested in the linear dynamics triggered by disturbances that are localized in space, in the physical sense, that is, when vorticity sources, perturbations on the interface and on the plate, and an initial perturbation in the flow are all negligibly small, for large $|x|$. Note that the stream function $\Psi$ is not necessarily localized in space, for every given localized-in-space velocity field $(u, v)$. In order to account for the localization assumption the governing equation (2.4) and the conditions (2.6)-(2.8) can be written in terms of the velocity component $v$ by differentiating each of them with respect to $x$. The corresponding perturbations of the resulting problem are localized in space. We arrive, therefore, at the formulation of the perturbed initial-value problem (IVP) for $v=v(y, x, t)$ as follows:

$$
\begin{aligned}
& {\left[R\left(\frac{\partial}{\partial t}+U \frac{\partial}{\partial x}\right) \nabla^{2}-R U^{\prime \prime} \frac{\partial}{\partial x}-\nabla^{4}\right] v=R G(y, x, t),} \\
& 0<y<1, \quad-\infty<x<\infty, \quad t>0, \\
& v(y, x, 0)=v_{0}(y, x), \quad 0 \leqslant y \leqslant 1, \quad-\infty<x<\infty, \\
& \left(\frac{\partial}{\partial t}+\frac{\partial}{\partial x}\right)\left(\frac{\partial^{2} v}{\partial y^{2}}-\frac{\partial^{2} v}{\partial x^{2}}\right)+2 \frac{\partial v}{\partial x}=f_{1}(x, t) \text { at } y=0, \\
& \frac{\partial^{3} v}{\partial y^{3}}+\left(\cot \theta \frac{\partial}{\partial x}-W \frac{\partial^{3}}{\partial x^{3}}\right)\left(\frac{\partial^{2} v}{\partial y^{2}}-\frac{\partial^{2} v}{\partial x^{2}}\right) \\
& +3 \frac{\partial^{3} v}{\partial x^{2} \partial y}-R \frac{\partial}{\partial y}\left(\frac{\partial v}{\partial t}+\frac{\partial v}{\partial x}\right)=f_{2}(x, t) \quad \text { at } y=0, \\
& v(1, x, t)=f_{3}(x, t), \quad \frac{\partial v}{\partial y}(1, x, t)=f_{4}(x, t), \quad-\infty<x<\infty, \quad t>0 .
\end{aligned}
$$

The functions $G(y, x, t), v_{0}(x, t), f_{1}(x, t), f_{2}(x, t), f_{3}(x, t), f_{4}(x, t)$ in (2.9) represent externally imposed perturbations and are assumed to have finite support in $(x, t)$ in order to ensure convergence of the integrals appearing in the treatment. The assumption of finite support will be subsequently relaxed to include all functions for which the formalism goes through, in particular, the functions that are physically localized in space and oscillatory in time (Briggs 1964; Bers 1973; Brevdo 1988). A formal solution of the IVP (2.9) is given in Appendix A.

\section{Determination of the response to a localized disturbance}

We are interested in the long-time asymptotic behaviour of $v\left(y, x_{0}+V t, t\right)$ given in (A 26), which is the solution of the IVP (2.9) along the ray $x=x_{0}+V t$, in the unstable case. Since the integral in (A26) has an analogous form to that of the integral in (A 24), the evaluation of the asymptotics is similar, for all $V$. We sketch the procedure for evaluating the asymptotics for $V=0$, that is, the asymptotics of $v\left(y, x_{0}, t\right)$, when $t \rightarrow \infty$. In its present form, the procedure was developed in the plasma physics literature (Briggs 1964; Bers 1973) for studying absolute and convective instabilities and spatially amplifying waves. It is based on the analytic continuation of the inverse 
Fourier integral in (A 24)

$$
R\left(x_{0}, \omega\right)=\int_{-\infty}^{\infty} \frac{T(y, k, \omega)}{D(k, \omega)} \mathrm{e}^{\mathrm{i} k x_{0}} \mathrm{~d} k
$$

in the strip $\{\omega \mid 0 \leqslant \operatorname{Im} \omega \leqslant \sigma\}$ on the complex $\omega$-plane. If the continuation of $R\left(x_{0}, \omega\right)$ starting from the Bromwich contour $\{\omega \mid \operatorname{Im} \omega=\sigma\}$ down to and slightly below the real $\omega$-axis is possible then the solution $v\left(y, x_{0}, t\right)$ decays exponentially as $t \rightarrow \infty$. On the other hand, if the analytic continuation of the function $R\left(x_{0}, \omega\right)$ in this strip has singular points then each of these points contributes to the growth of $v\left(y, x_{0}, t\right)$.

Determination of the singular points of $R\left(x_{0}, \omega\right)$ located between the Bromwich contour and the real $\omega$-axis reduces eventually to analysing the multiple $k$-roots of the dispersion relation function $D(k, \omega)$, for $\omega$ with $0<\operatorname{Im} \omega<\sigma$, that satisfy the collision criterion as follows. Let $\left(k_{0}, \omega_{0}\right)$ be such a root. We assume for simplicity that it is a double root in $k$ of $D\left(k, \omega_{0}\right)=0$, which is the most common case. Then in the limit $\operatorname{Im}\left(\omega-\omega_{0}\right) \rightarrow \infty, \operatorname{Re}\left(\omega-\omega_{0}\right)=0$, the root $k_{0}$ splits into two roots $k=k_{1}(\omega)$ and $k=k_{2}(\omega)$, with one of the roots moving to the upper half and the other to the lower half of the complex $k$-plane. In other words, when $\omega$ moves from above the Bromwich contour along the vertical line down to $\omega_{0}$, which we denote by $\omega \searrow \omega_{0}$, the $k$-roots $k_{1}(\omega)$ and $k_{2}(\omega)$ originate in this movement on opposite sides of the real $k$-axis and collide at $k=k_{0}$, for $\omega=\omega_{0}$. This kind of a collision is called pinching of the deformed Fourier contour because the function $R\left(x_{0}, \omega\right)$ cannot be continued analytically in $\omega=\omega_{0}$ by deforming the integration contour in (3.1), as it is 'pinched' from above and below by two $k$-roots that collide at $k=k_{0}$.

The dominant contribution to the asymptotics of the solution comes from the multiple $k$-root of $D(k, \omega)=0$ that satisfies the collision criterion and has maximum imaginary part of $\omega$ among all such roots. For $V \neq 0$, the same continuation procedure applies to the function

$$
R\left(x_{0}, \omega+V k\right)=\int_{-\infty}^{\infty} \frac{T(y, k, \omega+V k)}{D(k, \omega+V k)} \mathrm{e}^{\mathrm{i} k x_{0}} \mathrm{~d} k,
$$

and the multiple $k$-root of $D(k, \omega+V k)=0$ satisfying the collision criterion and possessing the largest $\operatorname{Im} \omega$ makes the dominant contribution to the asymptotics. Let, for the ray $x=x_{0}+V t$, this root be $(k(V), \omega(V))$. Then the asymptotics of the solution along this ray, as $t \rightarrow \infty$, is equal at the leading order to

$$
v\left(y, x_{0}+V t, t\right) \sim C(y, k(V), \omega(V)) t^{s} \mathrm{e}^{\omega_{i}(V) t} \mathrm{e}^{-\mathrm{i} \omega_{r}(V) t} \mathrm{e}^{-k_{i}(V) x_{0}} \mathrm{e}^{\mathrm{i} k_{r}(V) x_{0}},
$$

where the indices $r$ and $i$ denote the real and the imaginary parts, respectively. In (3.3), $C(y, k(V), \omega(V))$ is complex valued and independent of $t$ and $x_{0}$, and $s \in \mathbb{R}$ is a rational number that depends on the number and multiplicity of the colliding $k$-roots (cf. Brevdo 1988). In the case of two simple colliding roots, $s=-\frac{1}{2}$. The formula (3.3) is valid provided the roots of the numerator $T(y, k, \omega+V k)$ of the integrand in (3.2) do not cancel the contributing roots of the denominator $D(k, \omega+V k)$, for all $y \in[0,1]$ and for all admissible external perturbations. For the present flow no such cancellation of roots was found.

A search for multiple $k$-roots of $D_{V}(k, \omega) \equiv D(k, \omega+V k)=0$ that satisfy the collision criterion can be carried out by following the images of the horizontal lines

$$
\mathscr{L}_{\sigma}=\{\omega \mid \operatorname{Im} \omega=\sigma,-\infty<\operatorname{Re} \omega<\infty\}
$$

on the $k$-plane under the transformations $k=k_{n}(\omega), n=1,2, \ldots$, starting with $\sigma>\sigma_{m}$, 
and decreasing $\sigma$. Here $k=k_{n}(\omega), n=1,2, \ldots$, are the $k$-roots of $D_{V}(k, \omega)=0$, for a given $\omega$, and $\sigma_{m}$ is the maximum temporal growth rate, see (A 25). For $\sigma>\sigma_{m}$, when the line $\mathscr{L}_{\sigma}$ is a Bromwich contour, images located on opposite sides of the real $k$-axes are identified, and collisions of such images for decreasing $\sigma$ are detected. The collision of images that takes place because of a collision of $k$-roots is characterized by a change of pattern of the colliding curves (see Brevdo 1988).

Note that at the point of collision $k_{0}=k\left(\omega_{0}\right)$ of two simple $k$-roots (which is the most common case) the function $\omega_{V}(k)$, satisfying $\omega_{V}\left(k_{0}\right)=\omega_{0}$ and $D_{V}\left(k, \omega_{V}(k)\right) \equiv 0$ in a vicinity of $k_{0}$, has a simple saddle point. That is, $\mathrm{d} \omega_{V}\left(k_{0}\right) / \mathrm{d} k=0, \mathrm{~d}^{2} \omega_{V}\left(k_{0}\right) / \mathrm{d} k^{2} \neq 0$, provided $\partial D_{V}(k, \omega) / \partial \omega \neq 0$ at $\left(k_{0}, \omega_{0}\right)$. Let $\omega(k)=\omega_{V}(k)+V k$ in a vicinity of $k_{0}$ where $\omega_{V}(k)$ is defined. Then, in this vicinity, $D(k, \omega(k)) \equiv 0$. Hence, the frequency $\omega$ as a function of $k$, obtained as an $\omega$-root of $D(k, \omega)=0$ close to $\left(k_{0}, \omega_{0}\right)$, satisfies $\mathrm{d} \omega /\left.\mathrm{d} k\right|_{k=k_{0}}=V$.

The movement of images in the $k$-plane can be followed by computing numerically images of a discrete dense set of points on $\mathscr{L}_{\sigma}$. For this purpose $k$-roots of $D(k, \omega+$ $V k)=0$ have to be computed for a discrete set of $\omega$ on $\mathscr{L}_{\sigma}$, which is a timeconsuming procedure because it requires solving a boundary-value problem for the Orr-Sommerfeld equation in which the wavenumber $k$ appears to the fifth power (see the operator $B_{2}$ in (A 3)). On the other hand, in the same problem the frequency $\omega$ appears linearly, so computing $\omega$-roots of the equation $D(k, \omega+V k)=0$, for a given $k$, requires considerably less computer time than computing its $k$-roots, for a given $\omega$. This fact has stimulated an application of simple techniques based on computing $\omega$ as a function of $k$, for distinguishing between absolute and convective instabilities. One of these techniques that has been viewed until now as fairly reliable is presented in Appendix B.

The technique is based on computing the saddle point of the most unstable ray $x=x_{0}+V_{g} t$ and following the movement of the saddle point in the complex $\omega$-plane when $V$ varies continuously starting with $V_{g}$. As a result one obtains an interval of ray velocities $V \in\left(V_{l}, V_{r}\right)$ for which the saddle point is unstable. (Here and further in the text, the subscripts $l$ and $r$ used with the variable $V$ denote the left and the right points of an interval of velocities, respectively. The subscript $r$ used with $k$ and $\omega$ denotes the real part.) It is then assumed that (i) the interval $\left(V_{l}, V_{r}\right)$ consists of unstable ray velocities, and (ii) for each ray velocity this procedure computes the maximum growth rate. The assumption (ii) implies that outside the interval $\left(V_{l}, V_{r}\right)$ all ray velocities are stable.

The first part of this assumption (i) can be substantiated. Indeed, the saddle point of $\omega_{V}(k)$ connected by continuity in $V$ with the saddle point $\left(k_{m r}, 0\right)$ from which the procedure starts satisfies the collision criterion due to continuity. Therefore, all $V$ in the interval $\left(V_{l}, V_{r}\right)$ are unstable ray velocities. The second part of the assumption (ii) asserts that the traced saddle point remains dominant, for all $V$. This may be correct in some cases. See, for instance, Simmons \& Hoskins (1979) and Brevdo (1988) where the stability analysis of the Eady model was performed using the saddle point approach and the collision criterion, respectively, with the same results. Also, the saddle point analysis applied in Brevdo (1995) to the Blasius boundary layer gave the interval of unstable rays $\left(V_{l}, V_{r}\right)$ that agreed well with the experimental results of Gaster \& Grant (1975). However, in general, this might not be the case. In the present investigation we applied both methods to the film flow and discovered considerable discrepancies of the results because the saddle point approach failed to recover a significant portion of the interval of unstable rays. This occurred due to a bifurcation of the contributing most unstable saddle point. The results are presented in the next section. 

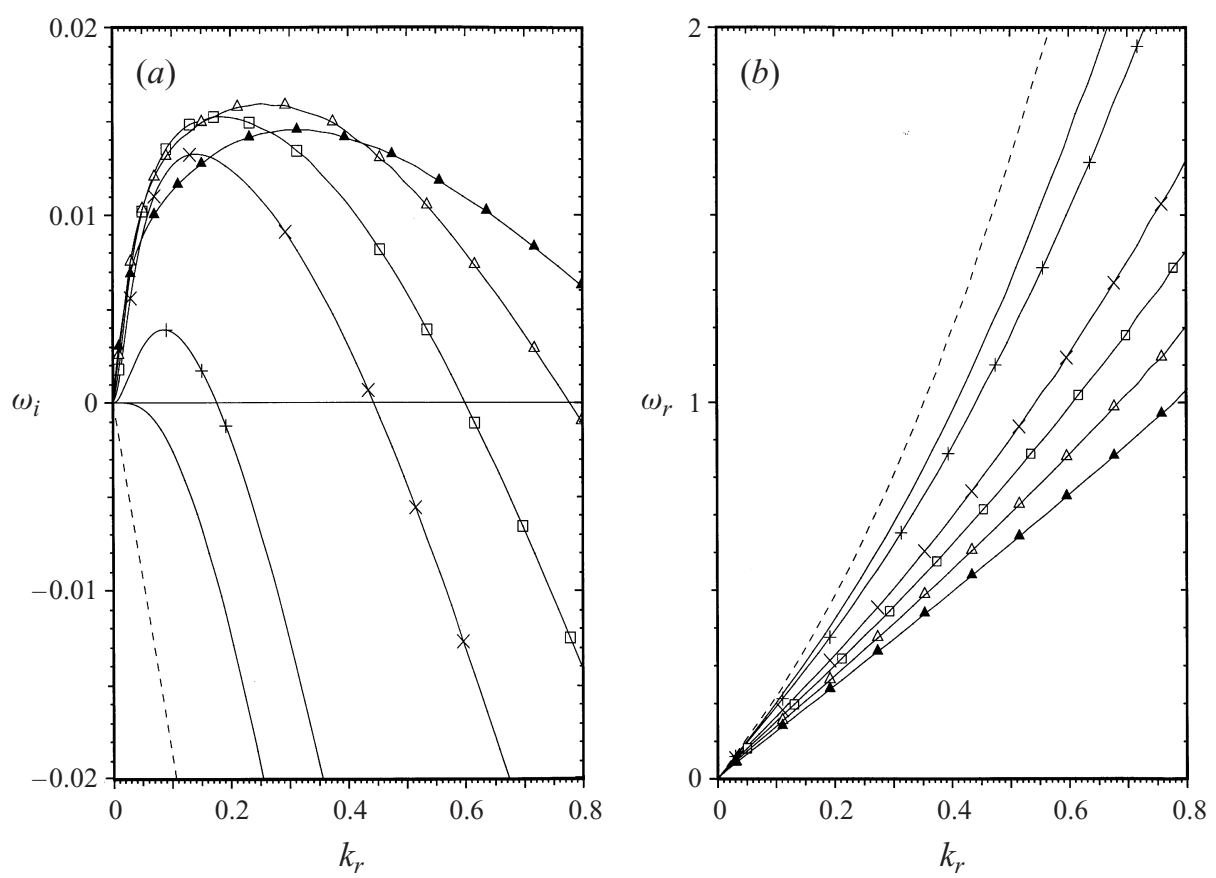

FIGURE 2. Most unstable branch of $\omega$ as a function of $k_{r}:(a)$ growth rate $\omega_{i},(b)$ angular frequency $\omega_{r}$. Values of the Reynolds number: $R=10(--), R_{c}(-), 20(+), 40(\times), 60(\square), 100(\triangle)$, and $200(\mathbf{\Delta})$. Values of the Weber number: $W=104.4(--), 77.88(-), 65.8(+), 41.46(\times), 31.6(\square)$, $22.5(\triangle)$, and $14.18(\boldsymbol{\Delta})$. Parameter values: $\theta=4.6^{\circ}, v=5.02 \times 10^{-6} \mathrm{~m}^{2} \mathrm{~s}^{-1}, T=69 \times 10^{-3} \mathrm{~N} \mathrm{~m}^{-1}$, and $\rho=1130 \mathrm{~kg} \mathrm{~m}^{-3}$.

\section{Convectively unstable wavepackets}

Before proceeding to the analysis of wavepackets we make some remarks concerning the temporal stability properties of the flow. Floryan, Davis \& Kelly (1987) have performed computations of the temporal stability of the flow for small angles and found out that, in this case, two unstable modes exist: a shear mode and a surface mode. For very small angles of less than about $0.5^{\prime}$, a destabilization of the flow occurs as a result of a shear mode instability. For angles greater than about $0.5^{\prime}$, a destabilization is caused by a surface mode instability, and for moderate unstable values of the Reynolds number no unstable shear mode is present. For the surface mode, in the long-wave limit $k=k_{r} \rightarrow 0$, one has

$$
\omega=2 k_{r}+\mathrm{i} \frac{2}{15}(-5 \cot \theta+4 R) k_{r}^{2}+o\left(k_{r}^{2}\right) .
$$

Hence, in this limit,

$$
R_{c}=\frac{5}{4} \cot \theta
$$

is the critical Reynolds number. Computations of Floryan et al. (1987) showed that $R_{c}$ given in (4.2) is the critical Reynolds number of the flow when destabilization is caused by a surface mode instability because such a destabilization occurs for infinitely long waves. In figure 2, computations of the unstable branch of $\omega$ as a function of $k_{r}$, presented for several values of the Reynolds number, illustrate this destabilization. The computations are performed by using a pseudo-spectral method to discretize the homogeneous boundary-value problem associated with the problem (A 2) (cf. Orszag 1971). The physical parameter values in figure 2 are those used in 
the experiments of Liu et al. (1993). The critical Reynolds number for the case shown in this figure is $R_{c}=15.54$. From figure 2(a), it is clearly seen that the growth rate $\omega_{i}$ satisfies quantitatively (4.1) in the unstable case only for very small $k_{r}$. Since the size of the interval of the unstable wavenumbers in this figure is of order one, for $R$ as low as 20 , the long-wave approximation is not suitable for investigating wavepacket asymptotics in this flow.

The analysis of wavepackets in this flow was performed using the approach based on the collision criterion. The results were compared to the results of a saddle-point treatment described in Appendix B. In the computations, we used the $\tau$-method and independently the Chebyshev collocation method applied for discretizing the homogeneous boundary-value problem associated with the problem (A 2) (cf. Orszag 1971). For solving the resulting algebraic eigenvalue problem in which $k$ appears to the fifth power, a companion matrix method was used (Bridges \& Morris 1984; Pearlstein \& Goussis 1988).

Most of the computations were carried out for the parameter values used in the experiments of Liu et al. (1993), see the caption of figure 2. At the same time other cases were also treated, including those for which stability computations were performed by Chang \& Demekhin (1996). In all unstable cases considered the film flow was found to be absolutely stable. In particular, we found that no transition from convective to absolute instability occurs for the value of the Reynolds number $R_{c / a}=R_{c}+(6.7 \mathrm{~W})^{1 / 3}$ predicted by the long-wave analysis of Benney (1966), where $R_{c}$ is the critical Reynolds number given by (4.2). For the experiments of Liu et al. (1993), $R_{c / a} \approx 23$. As already mentioned, the inadequacy of the long-wave approximation for the wavepacket analysis is due to the strong dispersion of waves with moderate wavelengths, see figure $2(a)$.

An illustration of the movement of the images of the lines $\mathscr{L}_{\sigma}$ under the transformations $k=k_{n}(\omega), D\left(k_{n}(\omega), \omega\right) \equiv 0, n=1,2, \ldots$, located close to the $k$-axis is given in figure 3. In figure 3(a), there are three images above and two images below the real $k$-axis, for $\sigma=\omega_{i}=0.02$. The image 1 that lies closest to the axis is shown to be above the axis in the close-up view in figure 3(b). In figure 3(c), for $\sigma=\omega_{i}=0$, and in the close-up view in figure $3(d)$, part of the image 1 is seen to cross the real $k$-axis, and there is no collision of images coming from opposite sides of the axis. Therefore, the flow is absolutely stable.

Computations of the growth rate $\omega_{i}(V)$, the oscillatory frequency $\omega_{r}(V)$, the local spatial amplification rate $-k_{i}(V)$ and the wavenumber $k_{r}(V)$ across the wavepacket were performed by using the collision criterion, for various values of the Reynolds number $R$. The results of the computations are presented in figure 4. For each Reynolds number $R=20,40$, and 60 , there is only one unstable branch of $\omega_{i}(V)$. In all these cases, the unstable branch is also recovered by using the procedure based on tracking the saddle point as described in Appendix B. However, for $R=100$ and 200 , the situation in this respect is dramatically different. We describe here in detail the case $R=200$ where the deviation of the results of the saddle point treatment from the results based on the collision criterion is more pronounced than in the case $R=100$.

For $R=200$, the analysis based on the collision criterion produces two unstable branches of $\omega_{i}(V)$, marked in figure $4(a)$ with $\boldsymbol{\Lambda}$ (branch I) and $\boldsymbol{\nabla}$ (branch II). The treatment based on the saddle point approach produces only one unstable branch of $\omega_{i}(V)$, branch I in figure $4(a)$. The second unstable branch, branch II, is not connected by continuity in $V$ with the saddle point corresponding to the most unstable ray. The same symbols are used in figure $4(b-d)$. The saddle point approach gives the 

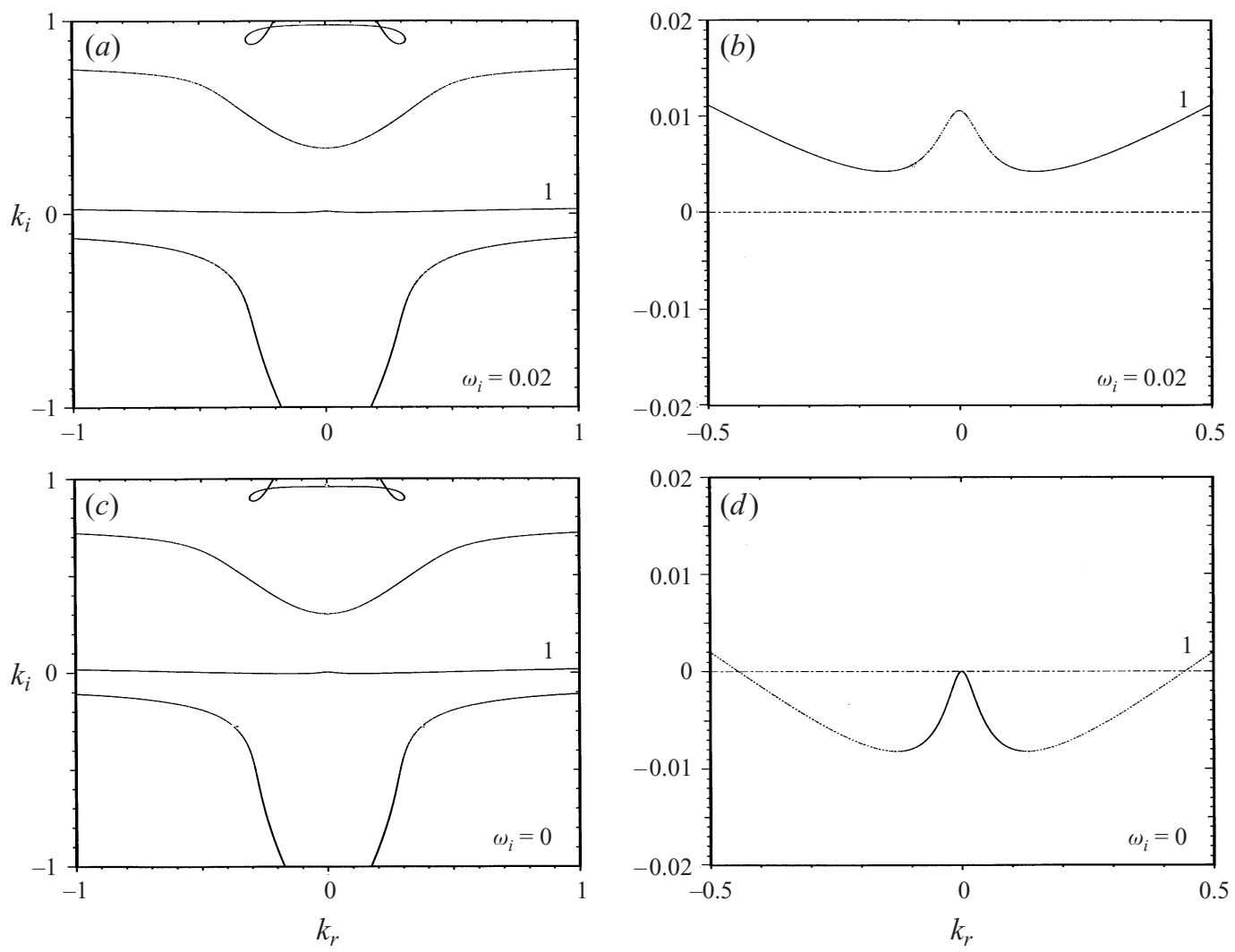

0
0
0
0
0
0
0
5
0
0
0
0
0
0
0
0
0
0
0
0
0
0
0
0
0

FIGURE 3. Movement of the images of the lines $\mathscr{L}_{\sigma}$ under the transformations $k=k_{n}(\omega), n=1,2, \ldots$, for $R=40$ See figure 2 for the other parameter values. 

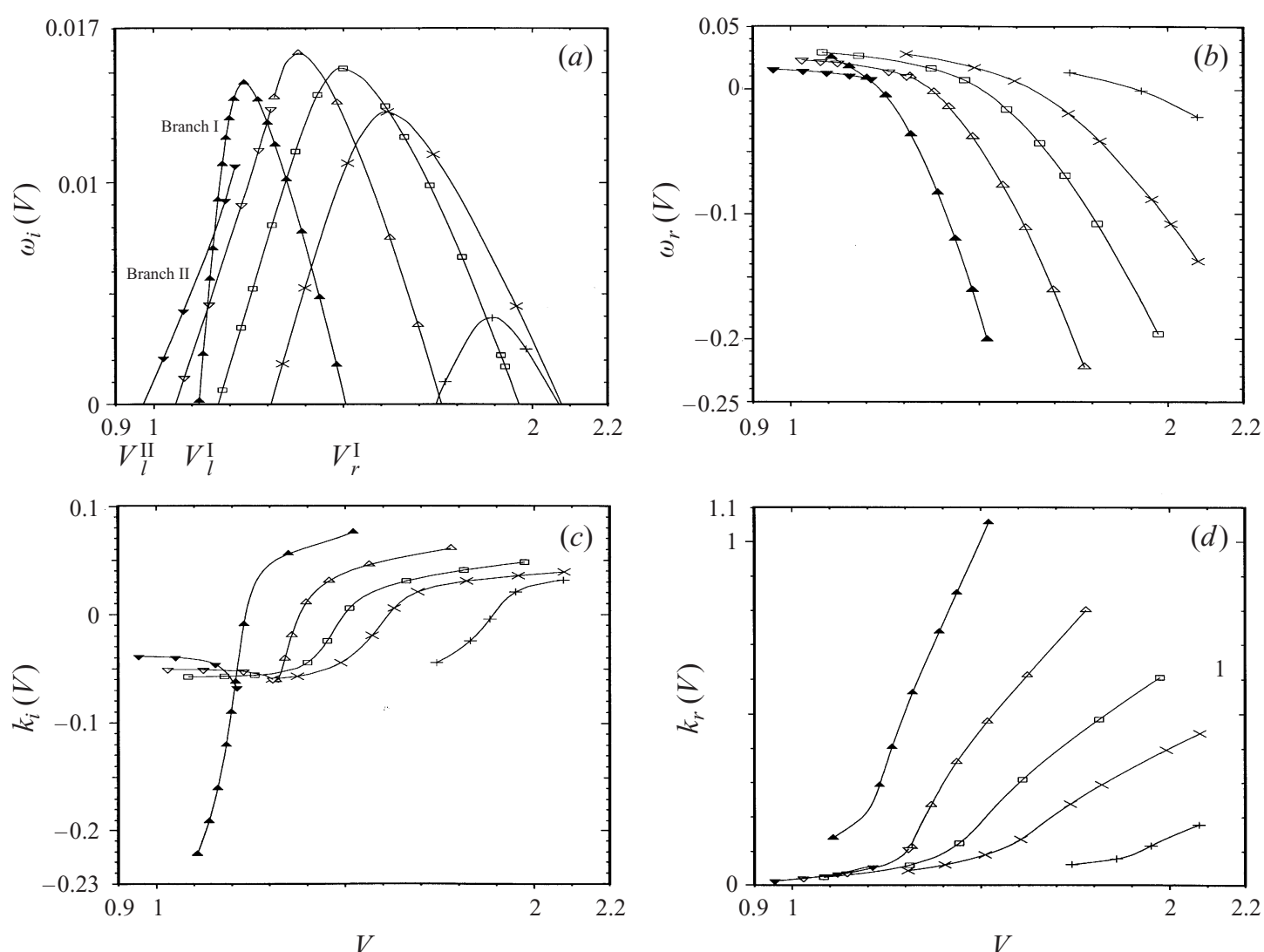

FIGURE 4. (a) Growth rate $\omega_{i}(V),(b)$ oscillatory frequency $\omega_{r}(V),(c)$ local spatial amplification rate with the sign minus $k_{i}(V)$, and $(d)$ wavenumber $k_{r}(V)$, as functions of the ray velocity $V$. Values of the Reynolds number are $R=20(+), 40(\times), 60(\square), 100(\triangle$ and $\nabla)$, and $200(\boldsymbol{\Delta}$ and $\boldsymbol{\nabla}$, referred to as branches I and II in the text). See figure 2 for the other parameters values. 
interval of unstable rays $\left(V_{l}^{I}, V_{r}^{I}\right)=(1.119,1.482)$, with the length $V_{r}^{I}-V_{l}^{I}=0.363$. The correct interval obtained using the collision criterion is $\left(V_{l}^{I I}, V_{r}^{I}\right)=(0.97,1.482)$, its length is $V_{r}^{I}-V_{l}^{I I}=0.512$. Therefore, the correct interval is more than $40 \%$ wider than the interval obtained with the saddle point treatment. Here the superscripts mark the branches, and, we recall, the subscripts $l$ and $r$ of $V$ correspond to the left and right points of the intervals, respectively. We see, therefore, that the saddle point treatment fails to recover the whole subinterval $\left(V_{l}^{I I}, V_{l}^{I}\right)=(0.97,1.119)$ of the interval of unstable rays of the length $V_{l}^{I}-V_{l}^{I I}=0.149$. Consequently, its prediction of the length of the unstable interval is made with a relative error of 0.29 , which is certainly not a negligible failure.

The two branches I and II correspond to collisions of images of the lines $\mathscr{L}_{\sigma}$ at different points in the $k$-plane, for the same value of $V$. This is demonstrated with two figures, figure 5 and figure 6 . Figure 5 is for $V=1.16$ which is slightly greater than the velocity $\widetilde{V}=1.1595$ at which the growth rates of both branches coincide, that is, $\omega_{i}^{I}(\widetilde{V})=\omega_{i}^{I I}(\widetilde{V})$. (See figure 7 for a location of $\widetilde{V}$.) For $\omega_{i}=0.01$, three images marked with 1, 2 and 3 participating in the collisions are shown in figure 5(a). Image 1 originates from above the real $k$-axis, while images 2 and 3 originate from below the axis, for $\omega_{i}=0.02$, which is greater than the maximum of $\omega_{i}$ for real $k$. In figure $5(b)$, for $\omega_{i}=0.0079$, image 1 coming from above the real $k$-axis collides with image 3 coming from below the real $k$-axis at two points symmetric with respect to the imaginary $k$-axis. One of these points is marked with $k^{I}$. It is a saddle point whose contribution lies on branch I in figure 4; it is connected by continuity in $V$ with the saddle point giving the contribution to the most unstable ray.

Following this collision there is a change of pattern of the images seen in figure $5(c)$, for $\omega_{i}=0.0078$. In this figure, another collision is seen. It is a collision of image 2 with a modified image 1 . Clearly, this collision occurs between $k$-roots originating on opposite sides of the real $k$-axis, because the colliding portion of the modified image 1 comes from above the real $k$-axis. A change of pattern of the images following this collision is seen in figure $5(d)$. One of the two points of collision having the same imaginary part is marked with $k^{I I}$. A contribution from this point belongs to branch II in figure 4. The saddle point $k^{I I}$ is not connected by continuity in $V$ with the saddle point of the most unstable ray. In figure 5(e) the trajectories of the colliding $k$-roots in the complex $k$-plane, when $\omega_{i}$ varies from $\omega_{i}=0.02$ down to $\omega_{i}=0$, are plotted for both collisions, with arrows indicating the movement of the roots. For each collision, the colliding roots originate on opposite sides of the real $k$-axis.

In figure 6 , the pinching process is shown for the ray velocity $V=1.15$ which is smaller than $\widetilde{V}$. Stages of the pinching process are shown in figure 6 in a manner similar to that of figure 5. This time, a collision of images 1 and 2 at the point $k^{I I}$ belonging to branch II in figure 4 occurs first in the process, for $\omega_{i}=0.0073$, and following it a collision at $k^{I}$ belonging to branch I takes place, for $\omega_{i}=0.0062$, see figures $6(b)$ and $6(c)$, respectively. The trajectories of the colliding $k$-roots in the complex $k$-plane plotted in figure $6(e)$ for $0.02 \geqslant \omega_{i} \geqslant 0$ show that, for each collision, the colliding roots originate on opposite sides of the real $k$-axis. Each branch I and II is making an additive contribution to the asymptotics of the wavepacket of the form (3.3). Hence, for the velocities $V$ in the interval $\left(V_{l}^{I}, V_{r}^{I I}\right)$ for which both branches are unstable, the asymptotics of the growing part of the wavepacket along rays with these velocities is a sum of two growing terms of the form (3.3), with $s=-\frac{1}{2}$.

A close-up view of branches I and II in figure 4 near the intersection point $\widetilde{V}$ of the curves $\omega_{i}=\omega_{i}^{I}(V)$ and $\omega_{i}=\omega_{i}^{I I}(V)$ is shown in figure 7. In each of figures 7(a)-7(d), a vertical dashed line is drawn for the ray velocity $V=\widetilde{V}$ at the intersection point, 
Pulse structure and signalling in a film flow on an inclined plane
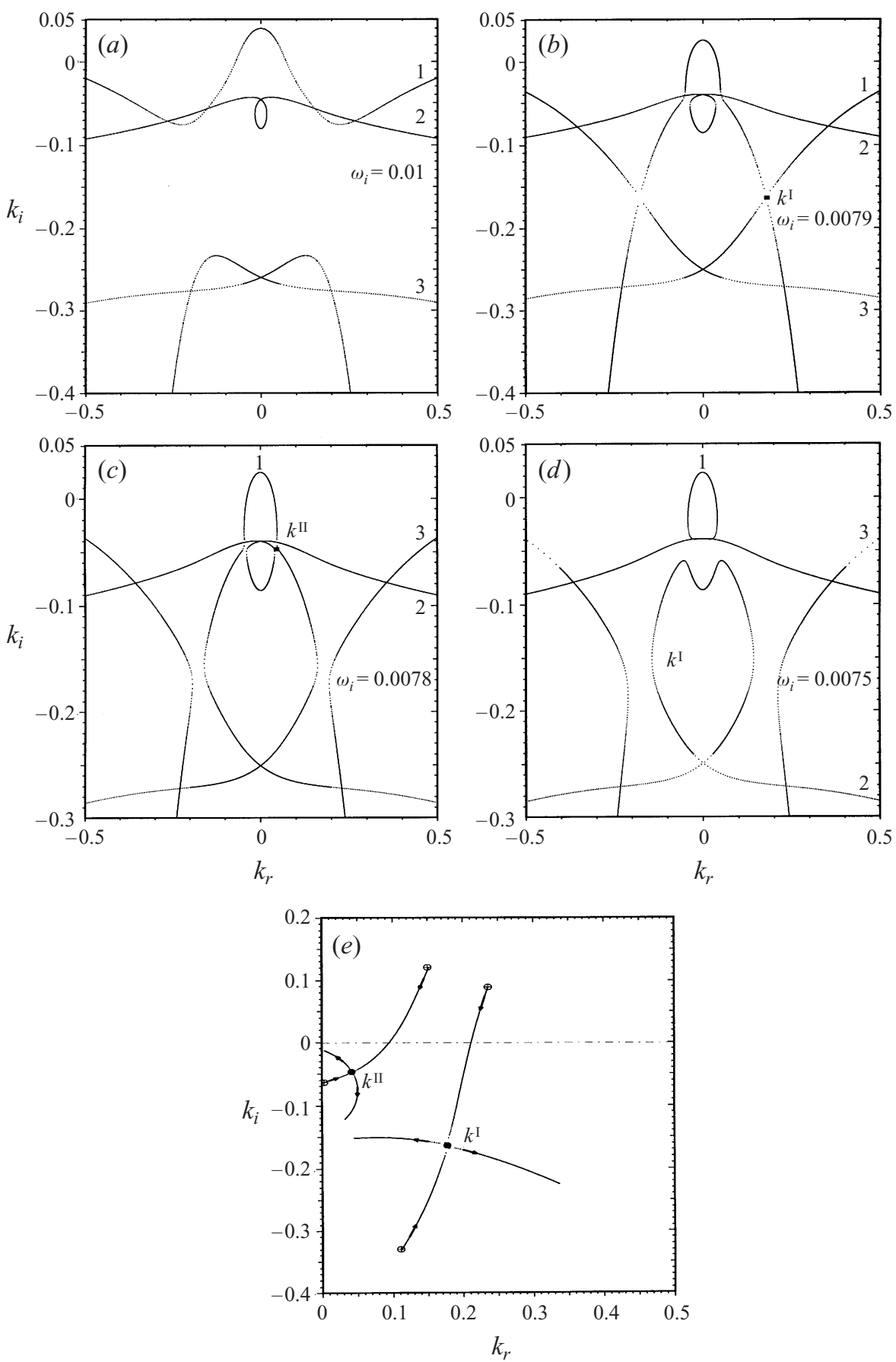

FIGURE 5. Pinching process in the complex wavenumber plane $\left(k_{r}, k_{i}\right)$ for $V=1.16, R=200$, $W=14.18$, and $\theta=4.6^{\circ}$ : (a) before the collisions; $(b)$ collision corresponding to branch $\mathrm{I}$ in figure $4, k^{I}=(0.19,-0.165)$ and the corresponding $\omega^{I}=(0.015,0.0079)$; $(c)$ collision corresponding to branch II in figure $4, k^{I I}=(0.045,-0.048)$ and the corresponding $\omega^{I I}=(0.015,0.0078)$; $(d)$ after the collisions; $(e)$ trajectories of the colliding $k$-roots for both collisions when $\omega_{i}$ decreases in both cases from 0.02 to 0 ; the arrows indicate the movement of the $k$-roots before and after the collision. 

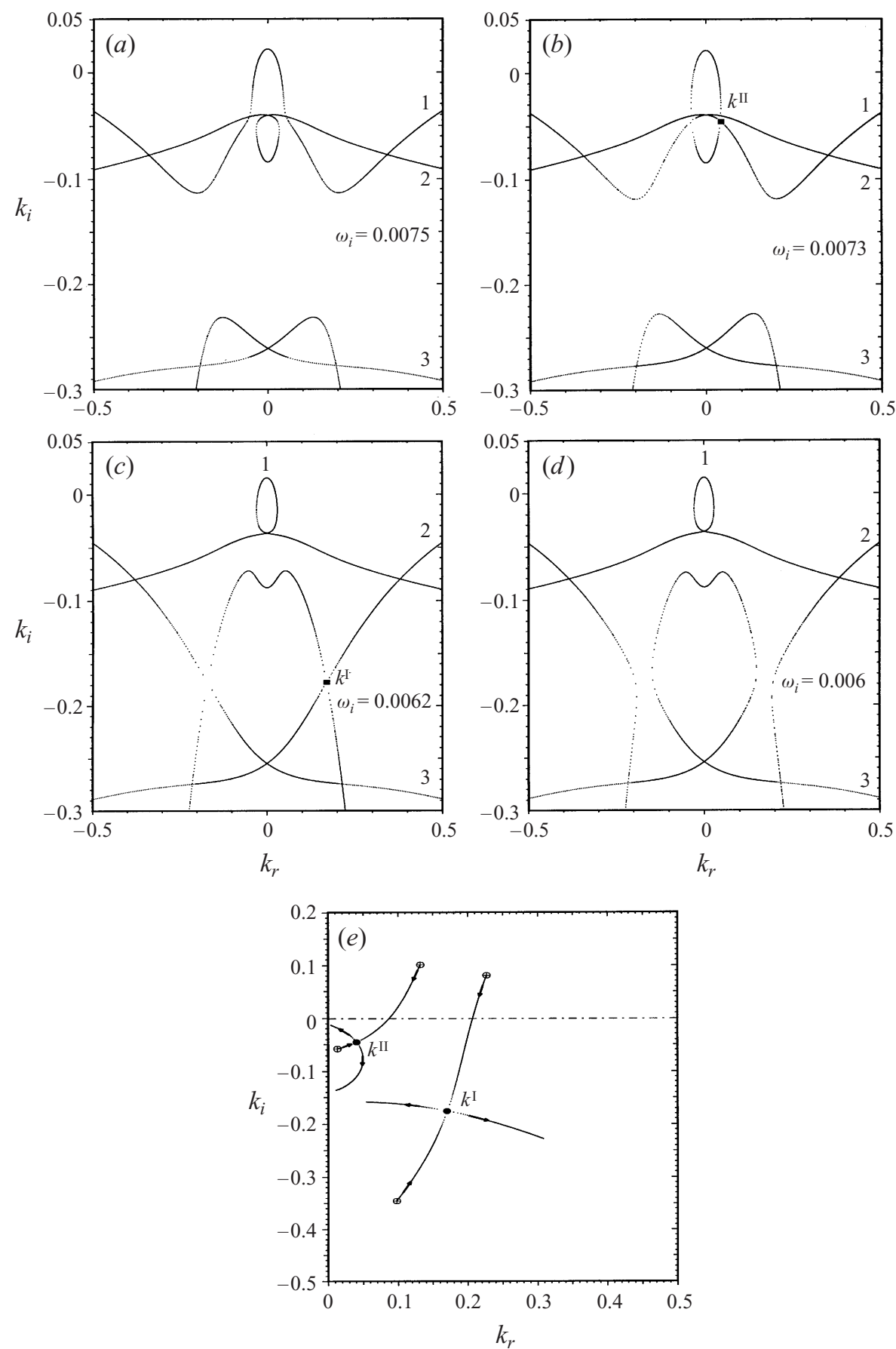

FIGURE 6. Pinching process in the complex wavenumber plane $\left(k_{r}, k_{i}\right)$ for $V=1.15, R=200$, $W=14.18$, and $\theta=4.6^{\circ}:(a)$ before the collisions; $(b)$ collision corresponding to branch II in figure $4, k^{I I}=(0.043,-0.046)$ and the corresponding $\omega^{I I}=(0.01,0.0073) ;(c)$ collision corresponding to branch $\mathrm{I}$ in figure $4, k^{I}=(0.17,-0.178)$ and the corresponding $\omega^{I}=(0.0182,0.0062)$; (d) after the collisions; $(e)$ trajectories of the colliding $k$-roots for both collisions when $\omega_{i}$ decreases in both cases from 0.02 to 0 ; the arrows indicate the movement of the $k$-roots before and after the collision. 

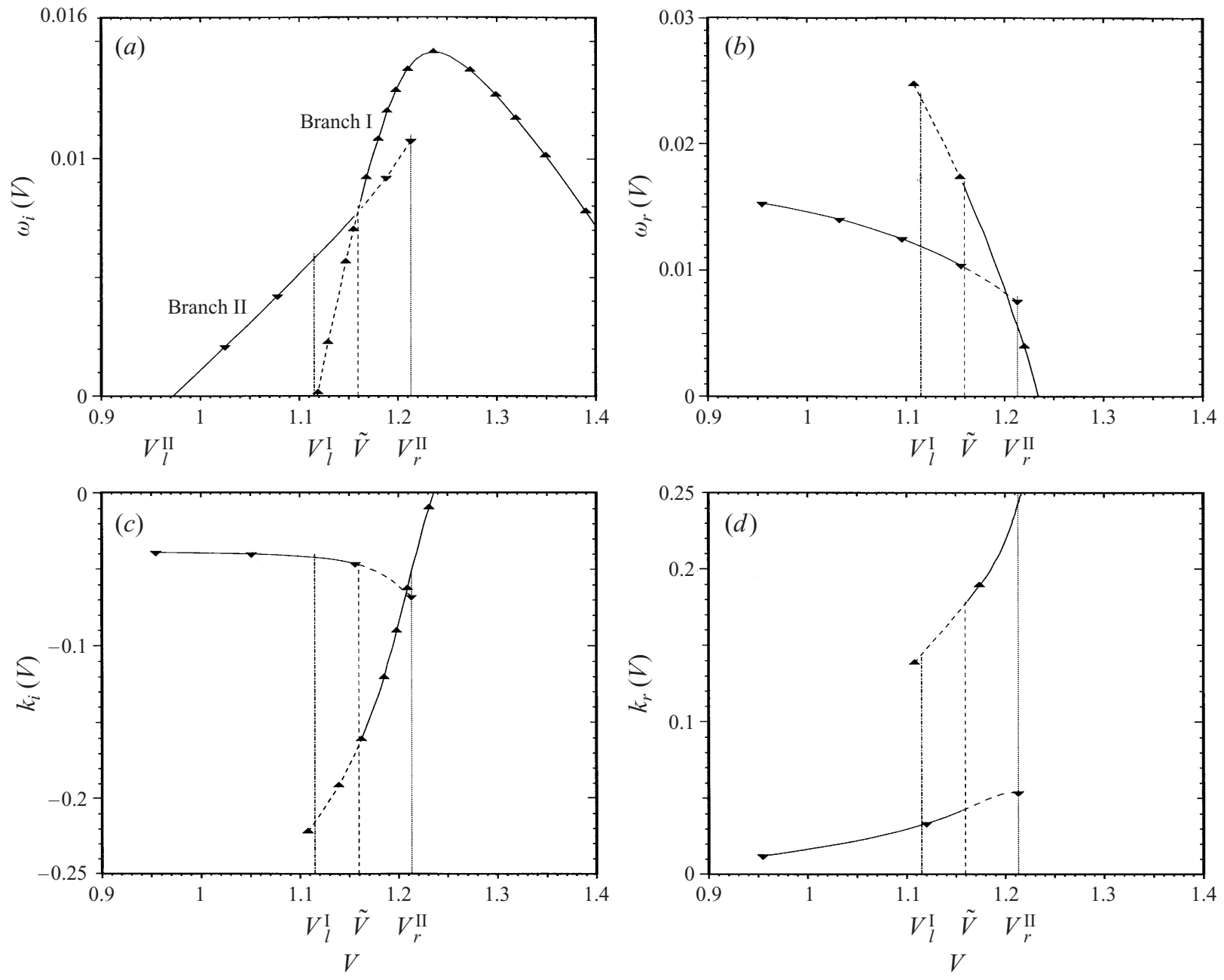

FIgURE 7. Close-up view of branches I $(\mathbf{\Lambda})$ and II $(\boldsymbol{\nabla})$ in figure 4 near the intersection point $\widetilde{V}$. The curves of the most unstable branch are drawn in continuous lines, the dashed lines give the subdominant branch. 
a vertical dashed-dotted line is drawn through the left margin $V_{l}^{I}$ of branch I, and a vertical dotted line is drawn through the right margin $V_{r}^{I I}$ of branch II. For $V>\widetilde{V}$, branch I is dominant, while for $V<\widetilde{V}$, branch II dominates the growth. In figures $7(a)-7(d)$, the curves representing the dominant branch are drawn in continuous lines, the curves of the subdominant branch are drawn in dashed lines.

At $V=\widetilde{V}$, the dominant growth rate $\omega_{i}(V)$ (figure $7 a$ ) is a continuous function of $V$. However, the corresponding oscillatory frequency $\omega_{r}(V)$ (figure $7 b$ ), the local spatial amplification rate with the minus sign $k_{i}(V)$ (figure $7 c$ ), and the wavenumber $k_{r}(V)$ (figure $7 d$ ), all of the dominant branch, are discontinuous at $V=\widetilde{V}$. In particular, the value of the wavenumber $k_{r}(V)$ has a jump at this point from about 0.175 on branch I to about 0.042 on branch II. This means that, for $V$ decreasing through $\widetilde{V}$, the local wavelength of the dominant part of the wavepacket $2 \pi / k_{r}(V)$ experiences at $V=\widetilde{V}$ an abrupt increase by a factor of about 4. For the values of $V$ in the interval $\left(V_{l}^{I}, \widetilde{V}\right)$, branch $\mathrm{I}$ is still unstable, though not dominant. Its wavelength is present in the growing wavepacket on this interval of $V$. However, on the interval of unstable $V$ to the left of $V_{l}^{I}$, i.e. on $\left(V_{l}^{I I}, V_{l}^{I}\right)$, there is only one unstable branch, branch II. On this interval the wavelength of branch I is no longer present in the growing part of the wavepacket, and the maximum wavenumber is about 0.033 . Therefore, as $V$ decreases starting with $\widetilde{V}$ down to and through $V_{l}^{I}$, the wavenumber of the growing asymptotics decays from 0.175 to 0.033 implying an increase of the wavelength on this interval of $V$ by a factor of about 5.3. We expect that such a strong increase of the wavelength across the interval of ray velocities $\left(V_{l}^{I}, \widetilde{V}\right) \approx(1.115,1.159)$ of the length of about 0.044 should be observable in experiments.

Interestingly, when $V$ increases starting with $V=\widetilde{V}$, branch II ceases to exist beyond the value of $V=V_{r}^{I I}$ of about 1.21 . We observe that such a situation is not possible, when $\omega(k)$ is analytic, because a branch of solutions of $\mathrm{d} \omega(k) / \mathrm{d} k=$ $V$ satisfying the collision criterion depends continuously on $V$ and hence cannot disappear beyond whatever $V$. Therefore, the reason for such a behaviour of branch II lies in the fact that, in the present case, $\omega=\omega(k)$ is a multi-valued function possessing branch point singularities in the $k$-plane. When $V$ increases towards $V_{r}^{I I}$, the point $k$ at which $\mathrm{d} \omega(k) / \mathrm{d} k=V$ holds for branch II moves towards such a singularity of $\omega=\omega(k)$, say $k=k_{r i g h t}^{I I}$, and reaches it for $V=V_{r}^{I I}$. As $V$ increases beyond $V_{r}^{I I}$, the equation $\mathrm{d} \omega(k) / \mathrm{d} k=V$ no longer has solutions lying on branch II and satisfying the collision criterion because the structure of the branches of the multi-valued function $\omega(k)$ changes at $k=k_{\text {right }}^{I I}$. This conclusion was substantiated by examining the patterns of the images of the lines $\mathscr{L}_{\sigma}$ in the $k$-plane, for $V$ to the left and to the right of $V_{r}^{I I}$. The point $V_{r}^{I I}$ appears as a bifurcation point, with $V$ being viewed as a bifurcation parameter. Indeed, for $V_{r}^{I}>V>V_{r}^{I I}$ there exists only one unstable branch, branch I. When $V$ decreases through $V_{r}^{I I}$ this first branch is present as before and in addition the second branch, II, appears. Also $V_{l}^{I}$ is a bifurcation point because there are two unstable branches, I and II, on the interval $V \in\left(V_{l}^{I}, V_{l}^{I}+\epsilon\right)$, where $\epsilon>0$ is small, while there is only one such branch, II, on the interval $V \in\left(V_{l}^{I}-\epsilon, V_{l}^{I}\right)$. We performed checks for the values of $V<V_{l}^{I I}$ and found no indication that branches I and II are connected where the saddle points have negative growth rates.

In figure 8 the loci of the contributing unstable saddle points in the $k$-plane, $k^{I, I I}(V)$, are shown for both branches I and II, for $V$ on the interval $\left[V_{l}^{I I}, V_{r}^{I}\right]$. The two loci are disconnected. From the point of view of the saddle point technique described in Appendix B we can deduce that, for each $V$ belonging to the interval 


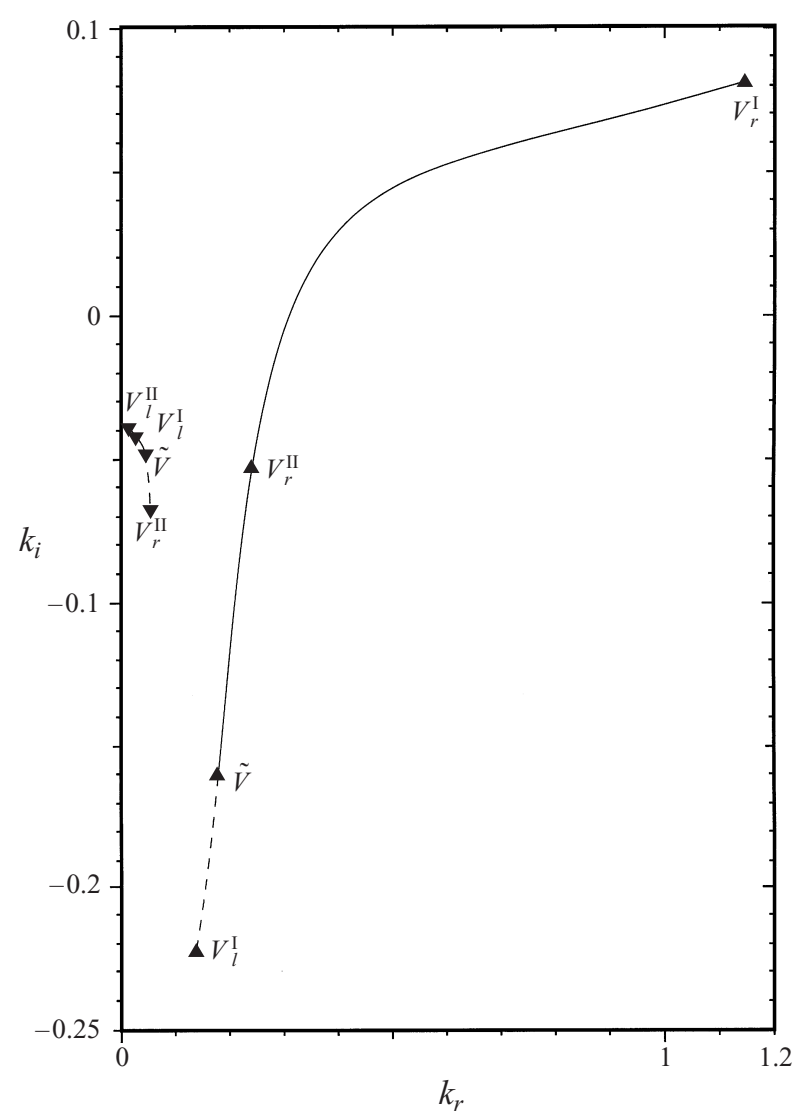

FIGURE 8. Loci of the unstable contributing saddle points in the $k$-plane, $k^{I, I I}(V)$, for both branches I - the curve on the right, and II - the curve on the left, as functions of $V$, for $V \in\left[V_{l}^{I I}, V_{r}^{I}\right] . R=200$. The dominant branch is drawn in continuous lines, the subdominant branch is drawn in dashed lines. See figure 2 for the other parameter values.

where both branches I and II are unstable, i.e. $V \in\left(V_{l}^{I}, V_{r}^{I I}\right)$, the equivalent steepestdescent contour passes through two contributing saddle points: one from branch I and the other one from branch II. Our computations show that at the saddle point of branch I the steepest descent contour is approximately parallel to the real $k$-axis, i.e. to the contour in (B 1). On the other hand, at the saddle point of branch II the steepest descent contour is approximately perpendicular to the contour in (B 1). This observation emphasizes that the orientation of the steepest descent path at a saddle point contains no information concerning the existence of the equivalent steepest-descent contour.

\section{Spatially amplifying waves}

Since the film flow on an inclined plane is absolutely stable for all values of the parameters considered, linear spatial patterns can be generated in it by applying a spatially localized periodic-in-time forcing with small amplitude. Such patterns in an absolutely stable but convectively unstable flow have the form of spatially amplifying waves that possess small amplitudes in a vicinity of the location of application $x=x_{0}$ of the periodic forcing and amplify exponentially with the distance $\left|x-x_{0}\right|$ from this 
location in the positive or negative $\left(x-x_{0}\right)$-direction. For sufficiently large $\left|x-x_{0}\right|$, the amplitude of a linear spatially amplifying wave becomes large, so that nonlinear effects take over and govern its further development in space. Secondary instabilities appear, and eventually transition to turbulence takes place at a certain distance from $x_{0}$. The structure of the spatially amplifying linear waves is important for understanding the evolution of secondary instabilities and for estimating the distance from $x_{0}$ to the area of development of a turbulent state.

\subsection{Formalism and admissible perturbations}

For studying spatially amplifying waves in the present flow, we assume that the component $v(y, x, t)$ is perturbed externally on the plate $y=1$, with all other external perturbations and the initial disturbance being zero. More general cases of external perturbations can be treated similarly. The only non-zero external perturbation is given by

$$
v(1, x, t)=f_{3}(x, t)=r\left(x-x_{0}\right) \mathrm{e}^{-\mathrm{i} \omega_{0} t},
$$

where $r(x)$ is a function with finite support, $x_{0}$ is fixed, and $\omega_{0} \in \mathbb{R}$ is the frequency of the forcing. This is a strictly harmonic-in-time perturbation. Forcing the flow by a perturbation of the form (5.1) is referred to as signalling. From (A 21)-(A 23) it follows that in this case

$$
\begin{aligned}
T(y, k, \omega) & =\frac{1}{\omega-\omega_{0}}(\omega-k)\left[v_{2}^{\prime}(1, k, \omega) v_{1}(y, k, \omega)-v_{1}^{\prime}(1, k, \omega) v_{2}(y, k, \omega)\right] r(k) \mathrm{e}^{-i k x_{0}} \\
& \equiv \frac{P(y, k, \omega) r(k)}{\omega-\omega_{0}} \mathrm{e}^{-\mathrm{i} k x_{0}},
\end{aligned}
$$

and the solution (A 24) takes the form

$$
v(y, x, t)=\frac{1}{4 \pi^{2}} \int_{\mathrm{i} \sigma-\infty}^{\mathrm{i} \sigma+\infty} \frac{\mathrm{e}^{-\mathrm{i} \omega t}}{\omega-\omega_{0}} \mathrm{~d} \omega \int_{-\infty}^{\infty} r(k) \frac{P(y, k, \omega)}{D(k, \omega)} \mathrm{e}^{\mathrm{i} k\left(x-x_{0}\right)} \mathrm{d} k .
$$

We do not have a proof of convergence of the integral in (5.3); and the difficulty here is that no perturbation-dependent function enters $P(y, k, \omega)$ that can be chosen in such a way as to obtain in a straightforward manner the desired asymptotics of the integrand at infinity in $\omega$ in the integration domain.

To assure convergence, we proceed like in Brevdo (1998) by assuming that the perturbation function $f_{3}(x, t)$ has the Fourier-Laplace transform of the form

$$
f_{3}(k, \omega)=\frac{A(k) B\left(\omega-\omega_{0}\right)}{\omega-\omega_{0}},
$$

where $A(k)$ is an entire function that decays rapidly at infinity on the real $k$-axis, and $B\left(\omega-\omega_{0}\right)$ decays rapidly at infinity in the strip $\left\{\omega \mid \omega \in \mathbb{C},-c<\operatorname{Im} \omega<\sigma_{m}\right\}$, for some positive $c$. Then the perturbation satisfies

$$
f_{3}(x, t)=A(x)\left[-\mathrm{i} B(0) \mathrm{e}^{-\mathrm{i} \omega_{0} t}+O\left(\mathrm{e}^{-\epsilon t}\right)\right],
$$

i.e. it is a slightly perturbed-in-time harmonic oscillator, with a deviation decaying exponentially for large $t$. Therefore, for large $t$, the external perturbation (5.5) can be 

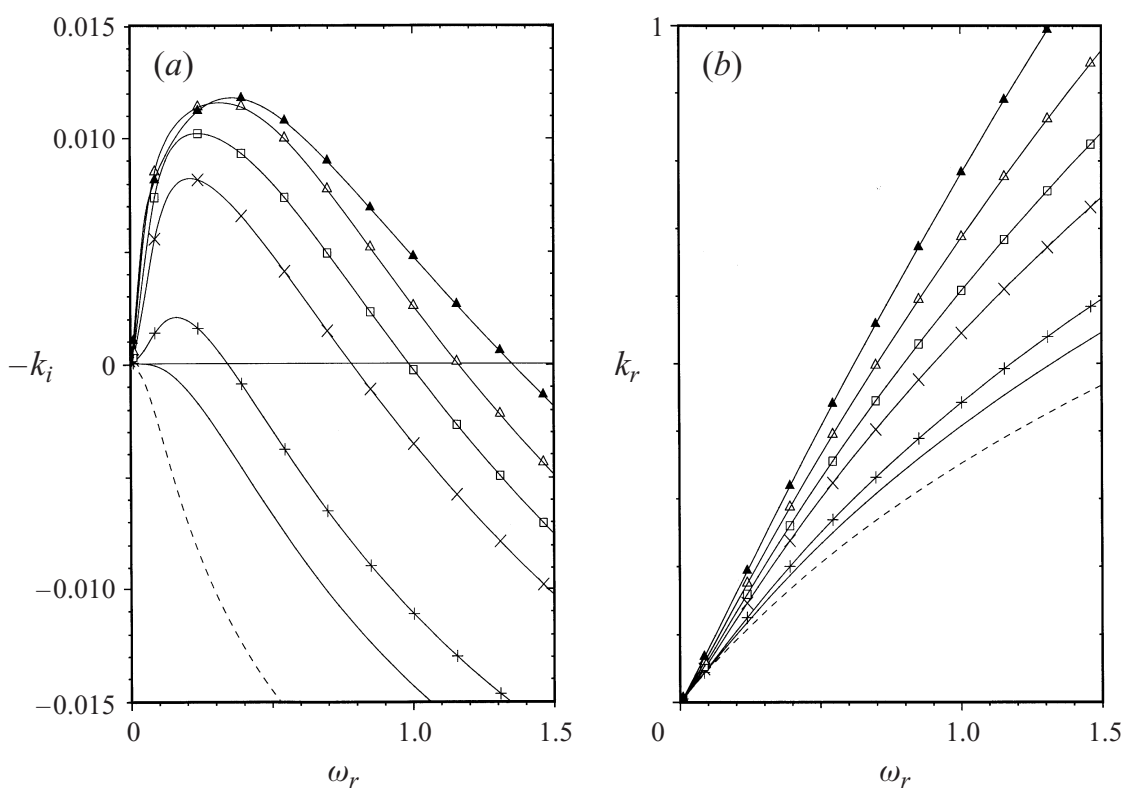

FIGURE 9. (a) Growth rate $-k_{i}$ and $(b)$ wavenumber $k_{r}$ of spatially amplifying waves as functions of signalling frequency $\omega_{r}$. The parameter values are: $\theta=4.6^{\circ}, v=5.02 \times 10^{-6} \mathrm{~m}^{2} \mathrm{~s}^{-1}$, $T=69 \times 10^{-3} \mathrm{~N} \mathrm{~m}^{-1}$ and $\rho=1130 \mathrm{~kg} \mathrm{~m}^{-3}$. Computations are done for $R=10(--), R_{c}(-), 20$ $(+), 40(\times), 60(\square), 100(\triangle)$, and $200(\mathbf{\Lambda})$. Values of the Weber number: $W=104.4(--), 77.88(-)$, $65.8(+), 41.46(\times), 31.6(\square), 22.5(\triangle)$, and $14.18(\mathbf{\Delta})$.

viewed for all purposes as harmonic in time. The solution is now given by

$$
v(y, x, t)=\frac{1}{4 \pi^{2}} \int_{\mathrm{i} \sigma-\infty}^{\mathrm{i} \sigma+\infty} \frac{B\left(\omega-\omega_{0}\right) \mathrm{e}^{-\mathrm{i} \omega t}}{\omega-\omega_{0}} \mathrm{~d} \omega \int_{-\infty}^{\infty} \frac{P(y, k, \omega) A(k)}{D(k, \omega)} \mathrm{e}^{\mathrm{i} k\left(x-x_{0}\right)} \mathrm{d} k,
$$

and the integral here can be made convergent by choosing $A(k)$ and $B\left(\omega-\omega_{0}\right)$ that possess the desired decay properties at infinity of the integration domain.

According to the mathematical formalism of spatially amplifying waves in the onedimensional case (Briggs 1964; Bers 1973), contributions to the growing asymptotics of the solution (5.6), for $t \rightarrow \infty,\left(x-x_{0}\right) \rightarrow \infty$, come from every $k$-root $k=k\left(\omega_{0}\right)$ of $D\left(k, \omega_{0}\right)=0$ that satisfies the following causality condition. For $\omega \searrow \omega_{0}$, the root $k=k(\omega)$ moves from above to below the real $k$-axis. This means that for $\omega$ above the Bromwich contour, the root is in the upper half of the $k$-plane; in the movement $\omega \searrow \omega_{0}$ the root crosses the real $k$-axis an odd number of times. Only $k$-roots crossing the real $k$-axis in this fashion contribute to the spatial amplification. For $t \rightarrow \infty,\left(x-x_{0}\right) \rightarrow-\infty$, contributions come from the $k$-roots that cross the real $k$-axis from below to above in the movement $\omega \searrow \omega_{0}$.

For the case $t \rightarrow \infty,\left(x-x_{0}\right) \rightarrow \infty$, let a contributing crossing root $k=k(\omega)$ reach a point $k_{0}$ at the end of its trajectory, when $\omega \searrow \omega_{0}$, i.e. $k_{0}=k\left(\omega_{0}\right)$. Then the contribution from this root to the asymptotics of the solution (5.6) in this case is given by

$$
C S\left(k_{0}\right)=C_{1}\left(y, k_{0}, \omega_{0}\right) \mathrm{e}^{\mathrm{i} k_{0}\left(x-x_{0}\right)} \mathrm{e}^{-\mathrm{i} \omega_{0} t},
$$

where $C_{1}\left(y, k_{0}, \omega_{0}\right)$ does not depend on $x$ and $t$. The $k$-root with maximum $\operatorname{Im} k\left(\omega_{0}\right)$ among all the crossing roots satisfying the causality condition makes the dominant 


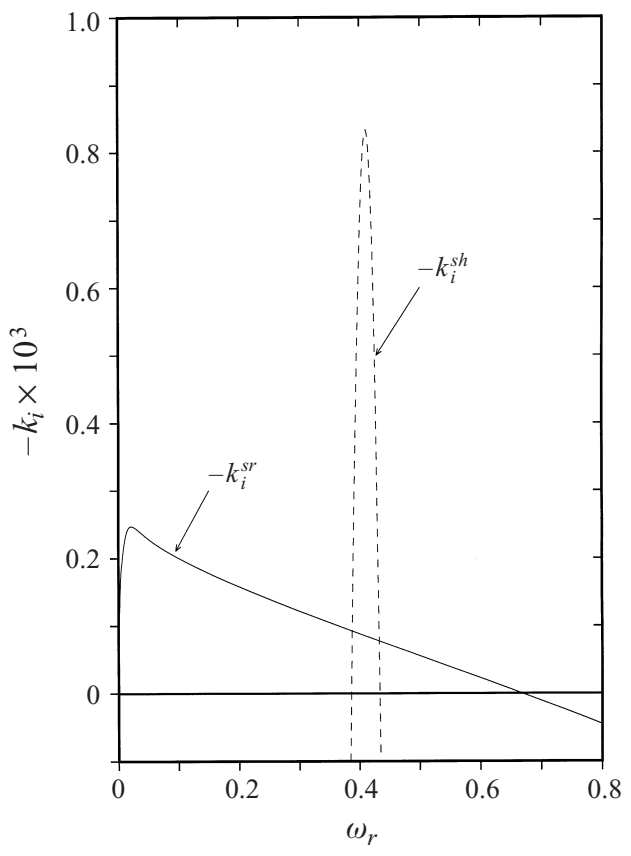

FIGURE 10. Growth rate $-k_{i}$ of spatially amplifying waves versus signalling frequency $\omega_{r}$ for $R=4100 ; s r$-surface mode branch, sh-shear mode branch. The parameter values are: $\theta=4^{\prime}$, $v=5.02 \times 10^{-6} \mathrm{~m}^{2} \mathrm{~s}^{-1}, T=69 \times 10^{-3} \mathrm{~N} \mathrm{~m}^{-1}$ and $\rho=1130 \mathrm{~kg} \mathrm{~m}^{-3}$.

contribution to the asymptotics, for $t \rightarrow \infty,\left(x-x_{0}\right) \rightarrow \infty$. For $t \rightarrow \infty,\left(x-x_{0}\right) \rightarrow-\infty$, the dominant contribution comes from the crossing root with maximum $-\operatorname{Im} k\left(\omega_{0}\right)$.

\subsection{Numerical results}

In the present case no spatially amplifying waves exist for $\left(x-x_{0}\right) \rightarrow-\infty$. For all supercritical Reynolds numbers there are spatially amplifying waves that amplify in the positive $x$-direction, i.e. when $\left(x-x_{0}\right) \rightarrow \infty$. Depending on the Reynolds number $R$ and the angle $\theta$ there are amplifying waves of the shear mode or of both the shear and the surface modes. In figure 9 the results of computations of the spatial growth rate $-k_{i}$ and the wavenumber $k_{r}$ as functions of signalling frequency $\omega_{r}=\omega_{0}$ are presented for several values of the Reynolds number. For the angle $\theta=4.6^{\circ}$ used in the computations the minimum critical Reynolds number of the shear mode over all $\Gamma$ is well above 1000 , i.e. well above the maximum Reynolds number $R=200$ in figure 9. Hence, spatially amplifying waves of only the surface mode are present. Neutral curves for the shear and surface modes for small values of the angle $\theta$ and different values of the Weber number (surface tension number) were computed by Floryan et al. (1987).

In terms of the expression (5.7), $\omega_{r}$ in figure 9 corresponds to $\omega_{0}$, and $k_{r}+\mathrm{i} k_{i}$ is $k_{0}$. For the case when the temporal and the spatial growth rates are small, the following transformation relating approximately these growth rates was proposed by Gaster (1962):

$$
-k_{i}(S)=\omega_{i}(T) / \frac{\partial \omega_{r}}{\partial k_{r}}
$$

where $S$ and $T$ mean that the values are obtained in the spatial and the temporal stability analyses, respectively. The Gaster transformation is widely used for com- 
puting spatial growth rates of normal modes by using computed temporal growth rates. This saves considerable computation time because spatial stability computations are much more time consuming than temporal stability ones. In Brevdo (1992b), a mathematical example was presented in which all the premises of the Gaster (1962) analysis were fulfilled but the formula (5.8) gave incorrect results, indicating that the Gaster transformation should be used with caution. Since in the present analysis we performed both the temporal and the spatial stability computations, we used the opportunity of checking the applicability of the Gaster formula (5.8). The outcome of this check is quite interesting. It turns out that the formula (5.8) not only gives a good approximation for the spatial growth rate when the growth rates are very small, but moreover, the dependence of the spatial growth rate given by (5.8) on the real part of frequency $\omega_{r}$ is practically indistinguishable from the results obtained in the direct spatial stability computations, for the entire range of unstable frequencies. In the present case, the Gaster transformation transforms the curves in figure 2(a) in the entire domain of unstable $k_{r}$ for all physical purposes practically identically into the corresponding curves in figure $9(a)$. Except for small vicinities of the points where the spatial growth rates in figure $9(a)$ are zero, the relative errors between the results computed by using the Gaster transformation and the results of the direct spatial stability computations are less than $10^{-3}$.

In figure 10 the spatial growth rate $-k_{i}$ is shown as a function of the signalling frequency $\omega_{r}$ for $R=4100$ and $\theta=4^{\prime}$. The notation is similar to that in figure 9 . This Reynolds number is greater than the critical Reynolds numbers for both the surface and the shear modes and, hence, two corresponding branches of amplifying waves are present. The surface mode branch is unstable for the source frequencies in the range $0<\omega_{r}<0.667$, whereas the maximum amplification rate $\max \left(-k_{i}^{s r}\right)=0.000247$ is attained at a rather low frequency $\omega_{r}=0.020$. The shear mode branch is unstable in a significantly narrower range $0.385<\omega_{r}<0.435$. The maximum amplification rate of this branch $\max \left(-k_{i}^{s h}\right)=0.000945$ attained at $\omega_{r}=0.41$ is almost four times greater than that of the surface mode branch. This maximum is ten times greater than the maximum amplification rate of the surface mode branch in the domain $0.385<\omega_{r}<0.435$ of unstable frequencies of the shear mode branch. This means that when the frequency of forcing $\omega_{r}$ increases starting with the left point of the domain $0.385<\omega_{r}<0.435$ towards the point $\omega_{r}=0.41$, i.e. increases by about $6.5 \%$, the growth rate of the most unstable spatially amplifying wave increases by a factor of about ten. We argue that such an abrupt increase of the growth rate should be observable in experiments if the flow can be kept laminar up to $R=4100$.

\subsection{Comparison with experiments}

Our computations are in fairly good agreement with the experimental results of Liu et al. (1993). In figure 11, we compare the computed growth rate and phase velocity with the measurements for $\theta=4.6^{\circ}, R=23$ and $W=62$. The growth rate in figure $11(a)$ is in good agreement with the experiments. In particular, the most unstable wavenumber and the cutoff wavenumber are correctly predicted. The shape of the phase velocity curve in figure $11(b)$ is similar to the distribution shape of the measurements with a deviation less than $2.5 \%$.

The comparison in figure 12(a) of the computed cutoff frequency with the measurements at $\theta=5.6^{\circ}$ shows good agreement. In figure $12(b)$ a comparison is made between the computed and measured cutoff frequencies, and between the computed and measured most rapidly amplified frequencies at $\theta=4.6^{\circ}$. Although there is a discrepancy between the cutoff frequencies, the shape delineated by the measurement 

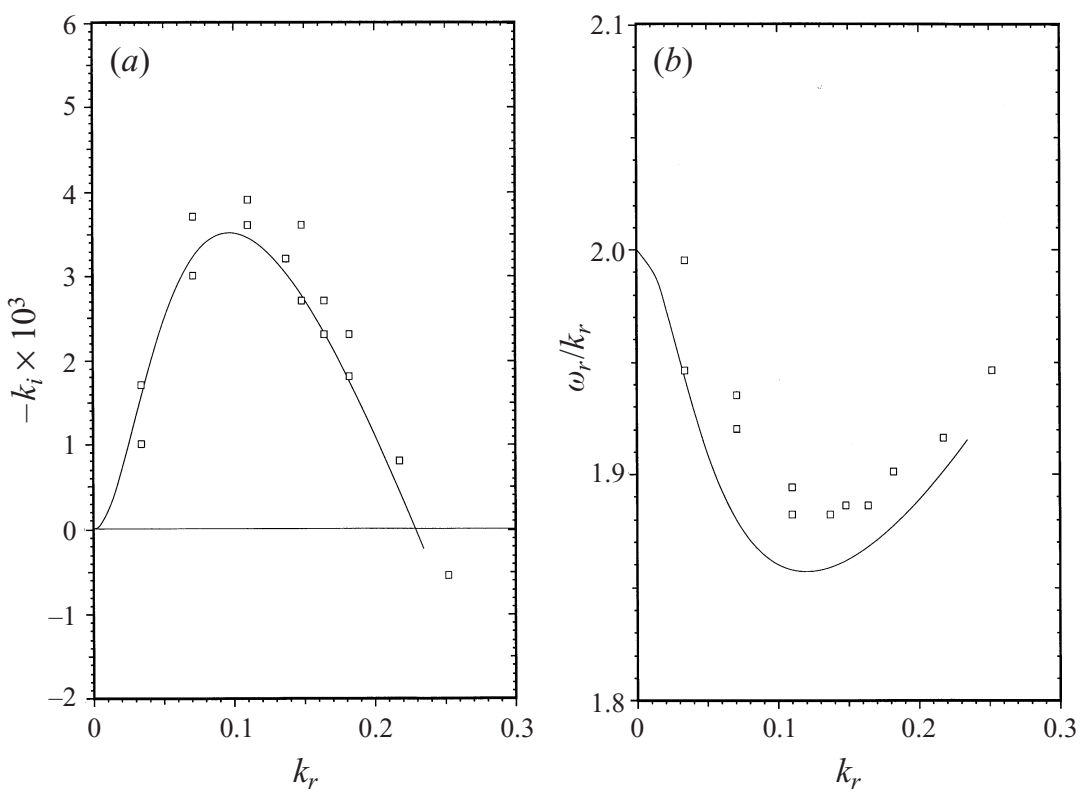

FIGURE 11. (a) Spatial growth rate $-k_{i}$ and $(b)$ phase velocity as functions of wavenumber $k_{r}$. Measurements $(\square)$ are taken from figure 14 in Liu et al. (1993). The parameter values are $\theta=4.6^{\circ}$, $v=4.89 \times 10^{-6} \mathrm{~m}^{2} \mathrm{~s}^{-1}, T=69 \times 10^{-3} \mathrm{~N} \mathrm{~m}^{-1}$ and $\rho=1130 \mathrm{~kg} \mathrm{~m}^{-3}$.

points parallels the shape of the computed curve. We attribute the discrepancy to a possible uncertainty while measuring a response to an oscillatory forcing when the amplification rate is very small. The most rapidly amplified frequencies compare well.

\section{Concluding remarks}

In this paper we have treated analytically and numerically the linear stability problem for localized disturbances in a film flow on an inclined plane. The formalism of wavepackets and spatially amplifying waves was implemented numerically. We have shown that, for a wide range of supercritical values of the Reynolds number relevant to experiments, the film flow on an inclined plane is convectively unstable but absolutely stable, and studied in detail the structure of unstable wavepackets, for a parameter case relevant to experiments. The convective nature of instability of the flow was established previously experimentally and supported by numerical results. However, no wavepacket analysis has been performed until now. Our investigation of the structure of the unstable wavepackets conducted by using both the Briggs collision criterion and the saddle-point approach has revealed that, in the case $R=200$, $\theta=4.6^{\circ}$, there is a bifurcation of the saddle-point contributing to the instability, where the velocity along the ray $V$ is a bifurcation parameter. The bifurcating saddle point, found by using the collision criterion, is not connected by continuity in $V$ with the contributing saddle point of the most unstable ray. We have studied the mathematical details and the physical implications of this bifurcation.

From the mathematical point of view, the appearance of a second contributing saddle point, as a result of the bifurcation, which is not connected by continuity in $V$ with the saddle point of the most unstable ray, means that the saddle-point treatment that relies on the continuity argument cannot be regarded as sound. The steepest- 

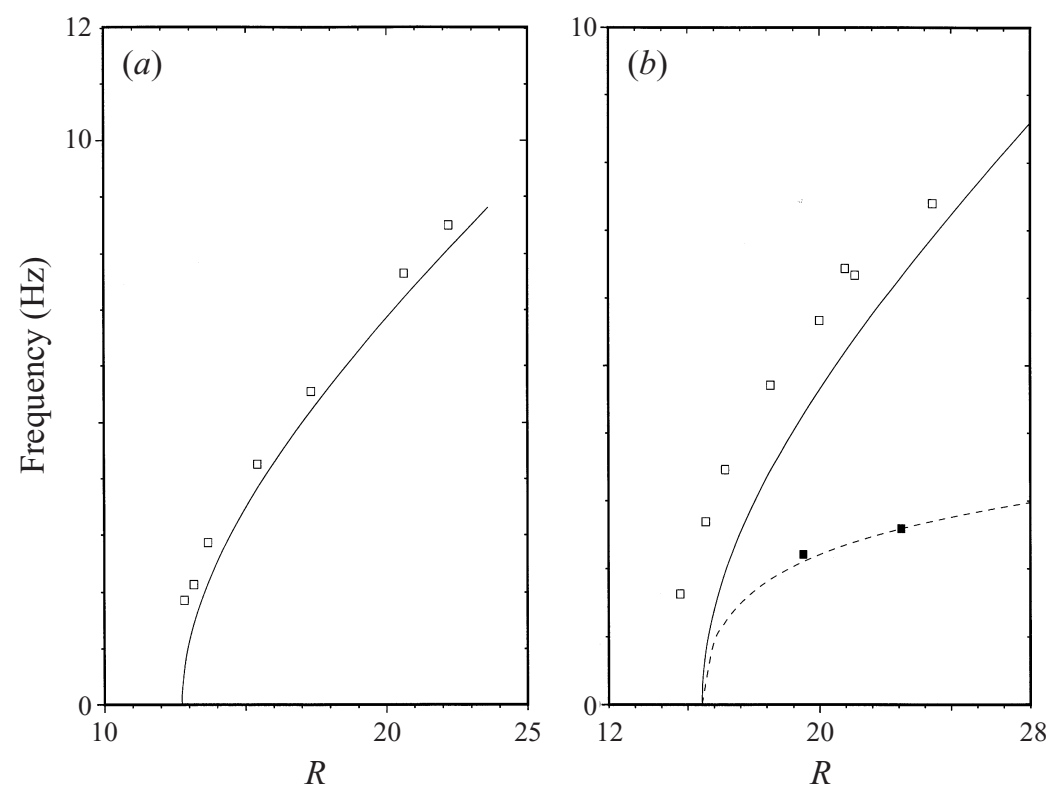

Figure 12. (a) Cutoff frequency $(-)$ for $\theta=5.6^{\circ}$ and $(b)$ cutoff frequency $(-)$ and most rapidly amplified frequency (- -) as functions of the Reynolds number $R$ for $\theta=4.6^{\circ}$. Experimental data, for cutoff frequency $(\square)$, and most rapidly amplified frequency $(\boldsymbol{\square})$, are taken from figures 4 and 18 in Liu et al. (1993). The parameters values are $v=5.02 \times 10^{-6} \mathrm{~m}^{2} \mathrm{~s}^{-1}, T=69 \times 10^{-3} \mathrm{~N} \mathrm{~m}^{-1}$ and $\rho=1130 \mathrm{~kg} \mathrm{~m}^{-3}$.

descent path at the saddle point connected by continuity in $V$ with the saddle point of the most unstable ray is almost parallel to the original Fourier contour, i.e. to the real $k$-axis. On the other hand, the steepest-descent path at the second-bifurcatingsaddle point is almost perpendicular to the original Fourier contour. This clearly shows that the orientation of the steepest-descent path at an unstable saddle point contains in itself no information with regard to whether this point contributes to the instability.

For $R=200, \theta=4.6^{\circ}$, the saddle-point procedure based on the continuity argument has failed to recover a significant subinterval of the interval of the unstable rays in the present case of a real physical flow. The failure casts doubt on the applicability of this procedure to other flows, thus virtually depriving the instability theory of one of its tools that until now was viewed as reliable. The implications of this outcome for two-dimensional flows are not too grave. Indeed, in the two-dimensional case, the Briggs collision criterion can be readily implemented numerically, and the only price that one has to pay for the ultimate reliability of the analysis is that computations based on this criterion require more CPU time than saddle-point computations.

On the other hand, the situation with regard to the methodology of analysing unstable wave packets in the three-dimensional case is not as simple. The analytical collision criterion for the absolute/convective instability classification of three-dimensional flows was derived by Brevdo (1991). According to this criterion, a complex $\omega_{0}$, with Im $\omega_{0}>0$, contributes to absolute instability if and only if (in the most common case) two $l$-roots of the system

$$
D(k, l, \omega)=0, \quad \frac{\partial D(k, l, \omega)}{\partial k}=0
$$


coming from opposite sides of the real $l$-axis in the complex $l$-plane collide, when $\omega \searrow \omega_{0}$. Each of the colliding $l$-roots must be a coalescence root, i.e. it must satisfy a certain collision condition formulated in terms of $k$-roots of the equation $D(k, l, \omega)=0$. Here, $k$ and $l$ are wavenumbers in two independent spatial directions, $\omega$ is a frequency and $D(k, l, \omega)$ is the dispersion relation function. The three-dimensional collision criterion is considerably more involved than the two-dimensional one, and at the present time no numerical algorithm for implementing this criterion is available. All numerical investigations of three-dimensional wavepackets in fluid flows known to us were conducted until now by applying a saddle-point treatment that uses the continuity argument. Since such a treatment can in general no longer be regarded as reliable, the necessity of developing a numerical procedure for implementing the collision criterion in the three-dimensional case is emphasized. Without such a procedure and corresponding checks of numerical results obtained previously neither these results nor possible future computations based on a saddle-point treatment that makes use of the continuity argument can be safely relied upon.

From the physical standpoint, the bifurcation of the contributing saddle point in the case studied reveals a strong jump discontinuity of the wavenumber of the most unstable contribution to the wavepacket as a function of the bifurcation parameter $V$. We expect that this discontinuity should be observable in experiments.

We have also performed spatial stability computations and treated spatially amplifying waves in this flow. The computational results compare well with the measurements of Liu et al. (1993). The comparison of the results of the Gaster (1962) transformation with the results of our direct computations has shown that, in the present case, this transformation reproduces practically identically the directly computed spatial growth rates of all unstable normal modes. For $R=4100$ and $\theta=4^{\prime}$, we found an abrupt increase of the spatial amplification rate of the shear branch mode as a function of the source frequency. We argue that if the flow can be kept laminar up to $R=4100$ then such an increase should be observable in experiments.

With regard to secondary instabilities, the work of Lin (1974) showed that the nonlinear periodic states, bifurcating from the neutral curve for the primary instability, could be studied using a complex Ginzburg-Landau equation with the coefficients determined numerically. Therefore the results of Brevdo \& Bridges (1996) should be applicable to this model to determine if the unstable region is broken into two regions: one absolutely unstable and the other absolutely stable but convectively unstable.

\section{Appendix A. Formal treatment of the IVP}

We treat the IVP (2.9) formally by applying the Fourier transform $F$ with respect to $x$ and the Laplace transform $L$ with respect to time $t$ defined by

$$
\begin{aligned}
& F\{u\}(k)=\widehat{u}(k)=\int_{-\infty}^{\infty} u(x) \mathrm{e}^{-\mathrm{i} k x} \mathrm{~d} x, \quad u(x)=\frac{1}{2 \pi} \int_{-\infty}^{\infty} \widehat{u}(k) \mathrm{e}^{\mathrm{i} k x} \mathrm{~d} k, \\
& \left.L\{w\}(\omega)=\widetilde{w}(\omega)=\int_{0}^{\infty} w(t) \mathrm{e}^{\mathrm{i} \omega t} \mathrm{~d} t, \quad w(t)=\frac{1}{2 \pi} \int_{\mathrm{i} \sigma-\infty}^{\mathrm{i} \sigma+\infty} \widetilde{w}(\omega) \mathrm{e}^{-\mathrm{i} \omega t} \mathrm{~d} \omega .\right\}
\end{aligned}
$$

The analysis is similar to that used in Brevdo $(1988,1992 a)$ for treating the IVPs for a geophysical flow and plane Poiseuille flow, respectively. Application of the transforms (A 1) to the problem (2.9) results in a non-homogeneous boundary-value problem 
(BVP) for a non-homogeneous Orr-Sommerfeld (OS) equation

$$
\begin{gathered}
\mathrm{L} v(y, k, \omega) \equiv\left[\left(\frac{\mathrm{d}^{2}}{\mathrm{~d} y^{2}}-k^{2}\right)^{2}-\mathrm{i} R(U k-\omega)\left(\frac{\mathrm{d}^{2}}{\mathrm{~d} y^{2}}-k^{2}\right)+\mathrm{i} k R U^{\prime \prime}\right] v(y, k, \omega) \\
=-R G(y, k, \omega)-R\left(\frac{\mathrm{d}^{2}}{\mathrm{~d} y^{2}}-k^{2}\right) v_{0}(y, k) \equiv S(y, k, \omega), \quad 0<y<1, \\
\mathrm{~B}_{1} v(0, k, \omega)=\frac{1}{\omega-k}\left[\mathrm{i} f_{1}(k, \omega)+\left.\mathrm{i}\left(\frac{\mathrm{d}^{2}}{\mathrm{~d} y^{2}}+k^{2}\right) v_{0}(y, k)\right|_{y=1}\right] \equiv \frac{1}{\omega-k} g_{1}(k, \omega), \quad \text { A } 2 b f_{2}(k, \omega)-R \frac{\mathrm{d} v_{0}}{\mathrm{~d} y}(0, k) \equiv g_{2}(k, \omega), \\
\mathrm{B}_{2} v(0, k, \omega)=f_{3}(k, \omega), \quad \frac{\mathrm{d} v}{\mathrm{~d} y}(1, k, \omega)=f_{4}(k, \omega)
\end{gathered}
$$

where the boundary condition operators $\mathrm{B}_{1}$ and $\mathrm{B}_{2}$ are given by

$$
\left.\begin{array}{l}
\mathrm{B}_{1}=\frac{\mathrm{d}^{2}}{\mathrm{~d} y^{2}}+k^{2}-\frac{2 k}{\omega-k}, \\
\mathrm{~B}_{2}=\frac{\mathrm{d}^{3}}{\mathrm{~d} y^{3}}+\mathrm{i} k\left(\cot \theta+W k^{2}\right)\left(\frac{\mathrm{d}^{2}}{\mathrm{~d} y^{2}}+k^{2}\right)-\left(\mathrm{i} k R-\mathrm{i} \omega R+3 k^{2}\right) \frac{\mathrm{d}}{\mathrm{d} y} .
\end{array}\right\}
$$

In (A 2), (A 3) and further in the text the tilde and the hat denoting the transformed variables are omitted for convenience. The dependent variables are distinguished from their transforms by pointing out the independent variables. Observe that the functions $G(y, k, \omega), v_{0}(y, k)$, and $f_{i}(k, \omega), 1 \leqslant i \leqslant 4$, are entire functions of $(k, \omega)$ (the first two of them are for every $y \in[0,1])$, since they are Fourier-Laplace transforms of functions with finite support in $(x, t)$. Hence, so are the functions $S(y, k, \omega), g_{1}(k, \omega)$, and $g_{2}(k, \omega)$. The operator $\mathrm{B}_{1}$ in (A 3) is singular at $\omega=k$. In the analysis below we present a modification of the procedure in Brevdo $(1988,1992 a)$ for constructing a solution of the IVP which takes this singularity into account.

\section{A.1. Dispersion relation}

For $\omega \neq k$, let $v_{1}(y, k, \omega), v_{2}(y, k, \omega), v_{3}(y, k, \omega)$ and $v_{4}(y, k, \omega)$ denote the solutions of the homogeneous equation associated with the equation of the problem (A 2), i.e. the one with $S(y, k, \omega)=0$, that satisfy the following initial conditions:

$$
\begin{array}{lrrr}
v_{1}=1, & v_{1}^{\prime}=0, \quad \mathrm{~B}_{1} v_{1}=0, & \mathrm{~B}_{2} v_{1}=0 & \text { at } y=0, \\
v_{2}=0, & v_{2}^{\prime}=1, \quad \mathrm{~B}_{1} v_{2}=0, & \mathrm{~B}_{2} v_{2}=0 & \text { at } y=0, \\
v_{3}=0, & v_{3}^{\prime}=0, \quad v_{3}^{\prime \prime}=1, & v_{3}^{\prime \prime \prime}=0 & \text { at } y=1, \\
v_{4}=0, & v_{4}^{\prime}=0, \quad v_{4}^{\prime \prime}=0, & v_{4}^{\prime \prime \prime}=1 & \text { at } y=1,
\end{array}
$$

where the prime denotes $\mathrm{d} / \mathrm{d} y$. The solutions $v_{3}$ and $v_{4}$ exist for any complex $k$ and $\omega$ and are entire functions of $(k, \omega)$ because the coefficients in the OS equation (A 2) are entire functions of $(k, \omega)$. Operators $\mathrm{B}_{1}$ and $\mathrm{B}_{2}$ defined in (A 3$)$ are of the second and third order, respectively, with the highest derivatives having the coefficient unity. The 
term $2 k /(\omega-k)$ in the expression for the operator $\mathrm{B}_{1}$ in (A 3) is analytic everywhere except at $\omega=k$; the rest of the coefficients in $\mathrm{B}_{1}$ and all the coefficients in $\mathrm{B}_{2}$ in (A 3) are entire functions of $(k, \omega)$. Therefore, the functions $v_{1}$ and $v_{2}$ exist and are analytic for all complex $(k, \omega)$, with a possible exception at $\omega=k$.

For $\omega \neq k$, let

$$
V_{1}(y, k, \omega)=(\omega-k) v_{1}(y, k, \omega) .
$$

Since, according to the definition, $v_{1}(y, k, \omega)$ is a solution of the homogeneous OS equation $\mathrm{L} v_{1}(y, k, \omega)=0$ satisfying the initial conditions (A $\left.4 a\right)$, the function $V_{1}(y, k, \omega)$ is a solution of the initial-value problem

$$
\left.\begin{array}{l}
\mathrm{L} V_{1}(y, k, \omega)=0,0<y<1, \\
V_{1}(0, k, \omega)=\omega-k, \quad V_{1}^{\prime}(0, k, \omega)=0, \\
V_{1}^{\prime \prime}(0, k, \omega)=-k^{2}(\omega-k)+2 k, \\
V_{1}^{\prime \prime \prime}(0, k, \omega)=-2 \mathrm{i} k^{2}\left(\cot \theta+W k^{2}\right) .
\end{array}\right\}
$$

Here the initial conditions for $V_{1}$ and $V_{1}^{\prime}$ at $y=0$ follow from the first two conditions of (A $4 a$ ), the initial conditions for $V_{1}^{\prime \prime}$ and $V_{1}^{\prime \prime \prime}$ are obtained by using the first two of (A $4 a$ ) in the second two of (A $4 a$ ) and using the expressions (A 3 ) for $\mathrm{B}_{1}$ and $\mathrm{B}_{2}$. The problem (A 6) is non-singular for all complex $k$ and $\omega$, including $\omega=k$. Since the coefficients of the OS operator $L$ and the right-hand sides of the initial conditions in (A 6) are entire functions of $(k, \omega)$, so is the solution $V_{1}(y, k, \omega)$ of the problem (A 6), for all $y \in[0,1]$. Observe that, for $\omega=k$, the function $V_{1}(y, k, k)$ satisfies the initial-value problem

$$
\left.\begin{array}{l}
\mathrm{L}_{\omega=k} V_{1}(y, k, k)=0, \quad 0<y<1, \\
V_{1}(0, k, k) \quad=0, \quad V_{1}^{\prime}(0, k, k)=0, \\
V_{1}^{\prime \prime}(0, k, k) \quad=2 k, \quad V_{1}^{\prime \prime \prime}(0, k, k)=-2 \mathrm{i}^{2}\left(\cot \theta+W k^{2}\right),
\end{array}\right\}
$$

and is, therefore, different from zero on the entire interval $0 \leqslant y \leqslant 1$, for $k \neq 0$, with an exception of at most a finite set of points $\mathscr{Y}$ in $[0,1]$. This follows from the analyticity in $y \in[0,1]$ of the coefficients of the OS operator in (A 2). Consequently, for every $y \in[0,1] \backslash \mathscr{Y}$, the function $v_{1}(y, k, \omega)=V_{1}(y, k, \omega) /(\omega-k)$ has a non-removable singularity, as a function of $(k, \omega)$, at $\left.(k, \omega)\right|_{\omega=k}$ in $\mathbb{C}^{2}$.

The function $v_{2}(y, k, \omega)$ defined for $\omega \neq k$ satisfies the initial-value problem

$$
\left.\begin{array}{l}
\mathrm{L} v_{2}(y, k, \omega)=0, \quad 0<y<1, \\
v_{2}(0, k, \omega)=0, \quad v_{2}^{\prime}(0, k, \omega)=1, \\
v_{2}^{\prime \prime}(0, k, \omega)=0, \quad v_{2}^{\prime \prime \prime}(0, k, \omega)=\mathrm{i} k R-\mathrm{i} \omega R+3 k^{2} .
\end{array}\right\}
$$

The initial conditions in (A 8) are derived in a fashion similar to that used for the problem (A 6). The solution of the problem (A 8) is an entire function of $(k, \omega)$ implying that, for all $y \in[0,1]$, the function $v_{2}(y, k, \omega)$ can be analytically continued to all points of the set $\{(k, \omega) \mid \omega=k, k, \omega \in \mathbb{C}\}$, i.e. analytically continued to an entire function. 
For $\omega \neq k$, we introduce the notation

$$
\boldsymbol{\Phi}(y, k, \omega)=\left[\begin{array}{cccc}
v_{1} & v_{2} & v_{3} & v_{4} \\
v_{1}^{\prime} & v_{2}^{\prime} & v_{3}^{\prime} & v_{4}^{\prime} \\
v_{1}^{\prime \prime} & v_{2}^{\prime \prime} & v_{3}^{\prime \prime} & v_{4}^{\prime \prime} \\
v_{1}^{\prime \prime \prime} & v_{2}^{\prime \prime \prime} & v_{3}^{\prime \prime \prime} & v_{4}^{\prime \prime \prime}
\end{array}\right] .
$$

The Wronskian $\mathscr{W}=\operatorname{det} \boldsymbol{\Phi}(y, k, \omega)$ does not depend on $y$, since the coefficient of the third derivative of $v(y, k, \omega)$ in the OS operator in (A2) is zero. Therefore, we can write

$$
\widetilde{D}(k, \omega)=\operatorname{det} \boldsymbol{\Phi}(y, k, \omega) .
$$

By using the conditions (A 4) at $y=1$ in (A 9) we obtain

$$
\begin{aligned}
\widetilde{D}(k, \omega) & =\operatorname{det}\left[\begin{array}{llll}
v_{1} & v_{2} & 0 & 0 \\
v_{1}^{\prime} & v_{2}^{\prime} & 0 & 0 \\
v_{3}^{\prime \prime} & v_{4}^{\prime \prime} & 1 & 0 \\
v_{3}^{\prime \prime \prime} & v_{4}^{\prime \prime \prime} & 0 & 1
\end{array}\right]_{\mid y=1} \\
& =\left[v_{1}(y, k, \omega) v_{2}^{\prime}(y, k, \omega)-v_{1}^{\prime}(y, k, \omega) v_{2}(y, k, \omega)\right]_{\mid y=1} \\
& =\frac{1}{\omega-k}\left[V_{1}(y, k, \omega) v_{2}^{\prime}(y, k, \omega)-V_{1}^{\prime}(y, k, \omega) v_{2}(y, k, \omega)\right]_{\mid y=1} \\
& =\frac{1}{\omega-k} D(k, \omega),
\end{aligned}
$$

with

$$
D(k, \omega)=\left[V_{1}(y, k, \omega) v_{2}^{\prime}(y, k, \omega)-V_{1}^{\prime}(y, k, \omega) v_{2}(y, k, \omega)\right]_{\mid y=1},
$$

where the definition (A 5) was used. Since $V_{1}(y, k, \omega)$ and $v_{2}(y, k, \omega)$ are entire functions of $(k, \omega)$ for all $y \in[0,1]$, so is the function $D(k, \omega)$.

In order to show that $\widetilde{D}(k, \omega)$ is not an entire function of $(k, \omega)$, we compute it for $\omega=c k$, where $c \neq 1$ is fixed, in the limit $k \rightarrow 0$. For $\omega=c k$, the initial-value problem for $v_{1}$ has coefficients analytic in $k$ and $y$ because the only singular coefficient $2 k /(\omega-k)$ in (A 2$)$, (A 3) appearing in $\mathrm{B}_{1}$ is equal to the constant $2 /(c-1)$, for such $k$ and $\omega$. The function $v_{2}(y, k, \omega)$ is an entire function of $(k, \omega)$. Therefore, in the limit $k \rightarrow 0$, the functions $v_{1}(y, k, c k)$ and $v_{2}(y, k, c k)$ converge in $C^{1}[0,1]$ in $y$ to the solutions of the following initial-value problems:

$$
\left.\begin{array}{l}
\frac{\mathrm{d}^{4}}{\mathrm{~d} y^{4}} v_{1}(y, 0,0)=0, \quad 0<y<1, \\
v_{1}(0,0,0)=1, \quad v_{1}^{\prime}(0,0,0)=0, \quad v_{1}^{\prime \prime}(0,0,0)=\frac{2}{c-1}, \quad v_{1}^{\prime \prime \prime}(0,0,0)=0,
\end{array}\right\}
$$

and

$$
\left.\begin{array}{l}
\frac{\mathrm{d}^{4}}{\mathrm{~d} y^{4}} v_{2}(y, 0,0)=0, \quad 0<y<1, \\
v_{2}(0,0,0)=0, \quad v_{2}^{\prime}(0,0,0)=1, \quad v_{2}^{\prime \prime}(0,0,0)=0, \quad v_{2}^{\prime \prime \prime}(0,0,0)=0,
\end{array}\right\}
$$


respectively. We have

$$
v_{1}(y, 0,0)=\frac{1}{c-1} y^{2}+1, \quad v_{2}(y, 0,0)=y,
$$

and, therefore, from (A 11),

$$
\lim _{\omega=c k, k \rightarrow 0} \widetilde{D}(k, \omega)=\left(\frac{1}{1-c} y^{2}+1\right)_{\mid y=1}=\frac{2-c}{1-c} .
$$

Since the limit (A 15) depends on the number $c$, whose value is different from 1 but otherwise arbitrary, the function $\widetilde{D}(k, \omega)$ is not analytic at $(k, \omega)=(0,0)$. This also shows that $\widetilde{D}(k, \omega) \not \equiv 0$ in $\mathbb{C}^{2}$, and, hence, $D(k, \omega) \not \equiv 0$.

The function $D(k, \omega)$ can also be expressed by evaluating the Wronskian at $y=0$. We recall that the operators $B_{1}$ and $B_{2}$ in (A 3) are of the second and third order respectively, and are linear combinations of the differentiation operators, with the coefficient of the highest derivative being unity in each of them. Hence, by applying row operations to the determinant of $\boldsymbol{\Phi}(y, k, \omega)$ it can be brought to the form

$$
\widetilde{D}(k, \omega)=\operatorname{det}\left[\begin{array}{cccc}
v_{1} & v_{2} & v_{3} & v_{4} \\
v_{1}^{\prime} & v_{2}^{\prime} & v_{3}^{\prime} & v_{4}^{\prime} \\
B_{1} v_{1} & B_{1} v_{2} & B_{1} v_{3} & B_{1} v_{4} \\
B_{2} v_{1} & B_{2} v_{2} & B_{2} v_{3} & B_{2} v_{4}
\end{array}\right] .
$$

We evaluate (A 16) at $y=0$ by using the corresponding conditions in (A 4) and the explicit expression for $B_{1}$ in (A 3). It yields

$$
\begin{aligned}
\widetilde{D}(k, \omega)= & \operatorname{det}\left[\begin{array}{cccc}
1 & 0 & v_{3} & v_{4} \\
0 & 1 & v_{3}^{\prime} & v_{4}^{\prime} \\
0 & 0 & B_{1} v_{3} & B_{1} v_{4} \\
0 & 0 & B_{2} v_{3} & B_{2} v_{4}
\end{array}\right]_{\mid y=0} \\
= & {\left[B_{1} v_{3}(y, k, \omega) B_{2} v_{4}(y, k, \omega)-B_{2} v_{3}(y, k, \omega) B_{1} v_{4}(y, k, \omega)\right]_{\mid y=0} } \\
= & \frac{1}{\omega-k}\left\{\left[(\omega-k)\left(\frac{\mathrm{d}^{2}}{\mathrm{~d} y^{2}}+k^{2}\right)-2 k\right] v_{3} B_{2} v_{4}\right. \\
& \left.-\left[(\omega-k)\left(\frac{\mathrm{d}^{2}}{\mathrm{~d} y^{2}}+k^{2}\right)-2 k\right] v_{4} B_{2} v_{3}\right\}_{\mid y=0}=\frac{1}{\omega-k} D(k, \omega),
\end{aligned}
$$

with

$$
\begin{aligned}
D(k, \omega)= & \left\{\left[(\omega-k)\left(\frac{\mathrm{d}^{2}}{\mathrm{~d} y^{2}}+k^{2}\right)-2 k\right] v_{3} B_{2} v_{4}\right. \\
& \left.-\left[(\omega-k)\left(\frac{\mathrm{d}^{2}}{\mathrm{~d} y^{2}}+k^{2}\right)-2 k\right] v_{4} B_{2} v_{3}\right\}_{\mid y=0} .
\end{aligned}
$$

All terms of the expression for $D(k, \omega)$ in (A 18) are entire functions of $(k, \omega)$, since the functions $v_{3}(y, k, \omega)$ and $v_{4}(y, k, \omega)$, and the coefficients of $\mathrm{B}_{2}$ also are entire.

As in the treatment of similar problems in Brevdo $(1988,1992 a)$ we have the following alternative. 
Proposition. For any complex pair $(k, \omega)$, with $\omega \neq k$, either $v_{1}, v_{2}, v_{3}$ and $v_{4}$ defined in $(A 4)$ are linearly independent or there exists a non-trivial solution $\breve{v}(y, k, \omega)$ of the homogeneous BVP associated with the problem (A2), i.e. the one with $S=g_{1}=g_{2}=$ $f_{3}=f_{4}=0$.

Proof. Let us assume that $v_{1}, v_{2}, v_{3}$ and $v_{4}$ are linearly independent. Then det $\boldsymbol{\Phi} \neq 0$ and the general solution of the homogeneous OS equation is given by $v=c_{1} v_{1}+c_{2} v_{2}+c_{3} v_{3}+c_{4} v_{4}$, where $c_{i}, 1 \leqslant i \leqslant 4$, are independent of $y$. According to (A4), the solution $v$ satisfies the homogeneous boundary conditions associated with the boundary conditions of the BVP (A 2) if and only if

and

$$
\left.\begin{array}{rl}
{\left[\begin{array}{ll}
B_{1} v_{3} & B_{1} v_{4} \\
B_{2} v_{3} & B_{2} v_{4}
\end{array}\right]_{\mid y=0}\left[\begin{array}{l}
c_{3} \\
c_{4}
\end{array}\right]} & =\left[\begin{array}{l}
0 \\
0
\end{array}\right], \\
{\left[\begin{array}{ll}
v_{1} & v_{2} \\
v_{1}^{\prime} & v_{2}^{\prime}
\end{array}\right]_{\mid y=1}\left[\begin{array}{l}
c_{1} \\
c_{2}
\end{array}\right]} & =\left[\begin{array}{l}
0 \\
0
\end{array}\right] .
\end{array}\right\}
$$

From (A 11) and (A 18), the determinant of the matrix of each of the systems in (A 18) is equal to det $\boldsymbol{\Phi} \neq 0$. Hence, the only solution of (A 18) is $c_{1}=c_{2}=c_{3}=c_{4}=0$, i.e. no non-trivial $\breve{v}(y, k, \omega)$ exists. On the other hand, if $v_{1}, v_{2}, v_{3}$ and $v_{4}$ are linearly dependent then $\operatorname{det} \boldsymbol{\Phi}=0$, and each of the systems in (A 18) has non-trivial solutions. We choose $c_{1}=c_{2}=0$, and let $\left(c_{3}, c_{4}\right)$ be an arbitrary non-trivial solution of the first of (A 18). Then the solution of the homogeneous OS equation given by $\breve{v}(y, k, \omega)=c_{3} v_{3}(y, k, \omega)+c_{4} v_{4}(y, k, \omega)$ satisfies the homogeneous boundary conditions associated with the conditions of the BVP (A 2). Moreover, this $\breve{v}(y, k, \omega)$ is non-trivial, since, according to (A 4), it satisfies $\breve{v}^{\prime \prime}(1, k, \omega)=c_{3}$ and $\breve{v}^{\prime \prime \prime}(1, k, \omega)=c_{4}$. Hence, at $y=1,\left(\breve{v}^{\prime \prime}\right)^{2}+\left(\breve{v}^{\prime \prime \prime}\right)^{2}=c_{3}^{2}+c_{4}^{2} \neq 0$.

An immediate consequence of the proposition is that the equation

$$
D(k, \omega)=0
$$

gives, for $\omega \neq k$, the complex dispersion relation of the problem as it is defined in the normal mode approach (Chandrasekhar 1961, pp. 3-6; Drazin \& Reid 1981, p. 11). Since $D(k, \omega)$ is analytic in $\mathbb{C}^{2}$, and $D(k, \omega) \not \equiv 0$, the set of solutions in $\omega$ of the equation $D\left(k_{0}, \omega\right)=0$ is, for every fixed complex $k_{0}$, either discrete or the entire complex plane. The latter is possible only when $D(k, \omega)=\left(k-k_{0}\right) Q(k, \omega)$, and $Q(k, \omega)$ is analytic in $\mathbb{C}^{2}$.

\section{A.2. Solution of the IVP}

Let $(k, \omega)$ be a complex pair, with $\omega \neq k$, which does not belong to the set of solutions of (A 19). Then the BVP (A 2) has a unique solution that can be found by using a variation of parameters, with the fundamental set of solutions $v_{i}, 1 \leqslant i \leqslant 4$, defined in (A 4). The solution is given by

$$
v(y, k, \omega)=\frac{T(y, k, \omega)}{D(k, \omega)},
$$

with

$$
T(y, k, \omega)=H(y, k, \omega)+\int_{0}^{1} K(y, \zeta, k, \omega) S(\zeta, k, \omega) \mathrm{d} \zeta
$$


where

$$
\begin{aligned}
H(y, k, \omega)= & (\omega-k)\left[v_{2}^{\prime}(1, k, \omega) f_{3}(k, \omega)-v_{2}(1, k, \omega) f_{4}(k, \omega)\right] v_{1}(y, k, \omega) \\
& +(\omega-k)\left[-v_{1}^{\prime}(1, k, \omega) f_{3}(k, \omega)+v_{1}(1, k, \omega) f_{4}(k, \omega)\right] v_{2}(y, k, \omega) \\
& +\left[B_{2} v_{4}(0, k, \omega) g_{1}(k, \omega)-(\omega-k) B_{1} v_{4}(0, k, \omega) g_{2}(k, \omega)\right] v_{3}(y, k, \omega) \\
& +\left[-B_{2} v_{3}(0, k, \omega) g_{1}(k, \omega)+(\omega-k) B_{1} v_{3}(0, k, \omega) g_{2}(k, \omega)\right] v_{4}(y, k, \omega),
\end{aligned}
$$

and

$K(y, \zeta, k, \omega)$

$$
=\left\{\begin{array}{l}
(\omega-k)\left[M_{41}(\zeta, k, \omega) v_{1}(y, k, \omega)-M_{42}(\zeta, k, \omega) v_{2}(y, k, \omega)\right] \text { for } y \leqslant \zeta, \\
(\omega-k)\left[-M_{43}(\zeta, k, \omega) v_{3}(y, k, \omega)+M_{44}(\zeta, k, \omega) v_{4}(y, k, \omega)\right] \text { for } y \geqslant \zeta .
\end{array}\right.
$$

In (A 23), $M_{m n}(\zeta, k, \omega)$ are the minors of the fundamental matrix $\boldsymbol{\Phi}$ defined in (A 9).

Recall that $V_{1}(y, k, \omega)=(\omega-k) v_{1}(y, k, \omega), v_{2}(y, k, \omega), v_{3}(y, k, \omega)$ and $v_{4}(y, k, \omega)$ are entire functions of $(k, \omega)$, for all $y \in[0,1]$. Hence, so are the functions $M_{41}(y, k, \omega)$, $(\omega-k) M_{42}(y, k, \omega),(\omega-k) M_{43}(y, k, \omega)$ and $(\omega-k) M_{44}(y, k, \omega)$, see (A 9). Consequently, $K(y, \zeta, k, \omega)$ is analytic in $(k, \omega)$ in $\mathbb{C}^{2}$, for all $y, \zeta \in[0,1]$. Since the functions $S(y, k, \omega)$, $f_{3}(k, \omega), f_{4}(k, \omega), g_{1}(k, \omega)$ and $g_{2}(k, \omega)$ are analytic in $(k, \omega)$ in $\mathbb{C}^{2}$, so is the function $H(y, k, \omega)$ for each $y \in[0,1]$. This implies that the function $T(y, k, \omega)$ is an entire function of $(k, \omega)$ for all $y \in[0,1]$.

By using (A 20) the solution of the IVP (2.9) can formally be expressed as

$$
v(y, x, t)=\frac{1}{4 \pi^{2}} \int_{\mathrm{i} \sigma-\infty}^{\mathrm{i} \sigma+\infty} \int_{-\infty}^{\infty} \frac{T(y, k, \omega)}{D(k, \omega)} \mathrm{e}^{\mathrm{i}(k x-\omega t)} \mathrm{d} k \mathrm{~d} \omega .
$$

Here $\sigma$ is a real number that satisfies

$$
\sigma>\sigma_{m}=\max \{\operatorname{Im} \omega \mid D(k, \omega)=0, \operatorname{Im} k=0\} .
$$

The flow is unstable when $\sigma_{m}>0$, and it is stable when $\sigma_{m} \leqslant 0$. For physical reasons, $\sigma_{m}$ must be finite because otherwise the problem is ill posed. Hence, $\sigma$ is positive in the unstable case. In the stable case, we can also choose $\sigma$ to be positive. Then in the entire integration domain of the integral in (A 24), $\omega \neq k$, since $k$ is real and Im $\omega=\sigma>0$. This implies that (A 24) indeed gives the solution of the IVP (2.9), provided the integral converges absolutely and uniformly in $y, x, t$, together with its corresponding derivatives appearing in (2.9).

In $\S \S 3,4$ we study the long-time asymptotics of the solution $v(y, x, t)$ along a ray $x=x_{0}+V t$, where $x_{0}$ is fixed and $V$ is the constant ray velocity. The solution (A 24) in this case can be expressed as

$$
\begin{aligned}
v\left(y, x_{0}+V t, t\right) & =\frac{1}{4 \pi^{2}} \int_{\mathrm{i} \sigma-\infty}^{\mathrm{i} \sigma+\infty} \int_{-\infty}^{\infty} \frac{T(y, k, \omega)}{D(k, \omega)} \mathrm{e}^{\mathrm{i}\left[k x_{0}-(\omega-V k) t\right]} \mathrm{d} k \mathrm{~d} \omega \\
& =\frac{1}{4 \pi^{2}} \int_{\mathrm{i} \sigma-\infty}^{\mathrm{i} \sigma+\infty} \int_{-\infty}^{\infty} \frac{T(y, k, \omega+V k)}{D(k, \omega+V k)} \mathrm{e}^{\mathrm{i}\left(k x_{0}-\omega t\right)} \mathrm{d} k \mathrm{~d} \omega .
\end{aligned}
$$

Since $T(y, k, \omega)$ is a linear combination (also in the sense of a continuous summation) of the functions $S(y, k, \omega), f_{3}(k, \omega), f_{4}(k, \omega), g_{1}(k, \omega)$ and $g_{2}(k, \omega)$ representing exter- 
nally imposed perturbations in (2.9), the convergence of integrals (A 24) and (A 26) can be achieved by imposing restrictions on perturbation functions in (2.9).

\section{Appendix B. Saddle-point approach}

The saddle-point approach in the two-dimensional case is derived from the following formal consideration (Briggs 1964; Bers 1973; Huerre 1987; Huerre \& Monkewitz 1985, 1990; Brevdo 1988). By applying the residue theory to the inverse Laplace integral in (A 26) the solution (A 26) can be expressed in the form

$$
v\left(y, x_{0}+V t, t\right)=\int_{-\infty}^{\infty} A(y, k) \mathrm{e}^{\mathrm{i}\left[k x_{0}-(\omega(k)-V k) t\right]} \mathrm{d} k .
$$

An asymptotic evaluation of the integral in (B 1) is carried out by using the steepestdescent method. For this purpose, the integration contour in (B 1) is deformed into an equivalent steepest-descent contour for the contributing saddle point of $\omega_{V}(k)=\omega(k)-V k$. Existence of the equivalent steepest-descent contour is the cornerstone question of the approach (Briggs 1964). The saddle-point approach in the three-dimensional case is similar. The collision criterion derived by Briggs (1964) allows one to look for contributing saddle points without addressing the existence of the global equivalent steepest-descent contour. Since checking the collision criterion directly in the two-dimensional case is numerically more expensive than searching for saddle points, and since no numerical procedure exists at present for checking the collision criterion for three-dimensional instabilities derived by Brevdo (1991), the saddle-point treatment was used in the literature for both cases.

The saddle-point technique in the two-dimensional case is based on computing points at which $\mathrm{d} \omega_{V} / \mathrm{d} k=0$ holds and $\operatorname{Im} \omega_{V}$ is positive. However, the existence of such points is not sufficient for instability, and to overcome this difficulty in the framework of the saddle-point treatment, a procedure described below, considered until now as reliable, was used in stability studies. We present here a description of this procedure for two-dimensional instabilities. In the three-dimensional case the procedure is similar.

The unstable branch of frequency $\omega(k) \equiv \omega_{r}\left(k_{r}, k_{i}\right)+\mathrm{i} \omega_{i}\left(k_{r}, k_{i}\right)$ is computed for real $k$. Note that $D(k, \omega(k)) \equiv 0$ holds. Let, for real $k, \omega_{i}\left(k_{r}, 0\right)$ attain its maximum at $k_{r}=k_{m r}$, and $V_{g}=\partial \omega_{r}\left(k_{m r}, 0\right) / \partial k_{r}$. The function $\omega_{V_{g}}(k)=\omega(k)-V_{g} k$ satisfies $D\left(k, \omega_{V_{g}}(k)+V_{g} k\right) \equiv 0$ and $\mathrm{d} \omega_{V_{g}}(k) / \mathrm{d} k=0$ at $k=k_{m r}$. Hence, $D\left(k, \omega+V_{g} k\right)=0$ has a double root in $k$ for $k=k_{m r}, \omega=\omega_{r}\left(k_{m r}, 0\right)-V_{g} k_{m r}+\mathrm{i} \omega_{i}\left(k_{m r}, 0\right)$. Moreover, the Taylor expansion of $\omega_{V_{g}}(k)$ at the point $k=k_{m r}$ is given by $\omega_{V_{g}}(k)=\omega_{V_{g}}\left(k_{m r}\right)+$ $\frac{1}{2} \mathrm{~d}^{2} \omega_{V_{g}}\left(k_{m r}\right) / \mathrm{d} k^{2}\left(k-k_{m r}\right)^{2}+O\left[\left(k-k_{m r}\right)^{3}\right]$. Therefore, if $\mathrm{d}^{2} \omega_{V_{g}}\left(k_{m r}\right) / \mathrm{d} k^{2} \neq 0$, which is the case in all instabilities known to us, the two colliding $k$-roots of $D\left(k, \omega+V_{g} k\right)=0$ that form the saddle point at $k_{r m}$ satisfy, to the leading order in $\left[\omega-\omega_{V_{g}}\left(k_{m r}\right)\right]$,

$$
k_{1,2}(\omega)=k_{m r} \pm \sqrt{2} \frac{\left[\omega-\omega_{V_{g}}\left(k_{m r}\right)\right]^{1 / 2}}{\left[\mathrm{~d}^{2} \omega_{V_{g}}\left(k_{m r}\right) / \mathrm{d} k^{2}\right]^{1 / 2}} .
$$

Consequently, $k_{1}(\omega)$ and $k_{2}(\omega)$ move to opposite sides of the real $k$-axis in the $k$ plane when $\omega$ moves vertically slightly starting with the point $\omega_{V_{g}}\left(k_{m r}\right)$. In the limit $\operatorname{Im}\left[\omega-\omega_{V_{g}}\left(k_{m r}\right)\right] \rightarrow \infty, \operatorname{Re}\left[\omega-\omega_{V_{g}}\left(k_{m r}\right)\right]=0$, each of these roots remains on the side of the real $k$-axis to which it originally moved because the imaginary part of $\omega_{V_{g}}(k)$ attains its maximum on the real $k$-axis at $k=k_{m r}$. This means that the roots $k_{1,2}(\omega)$ satisfy the collision criterion and, therefore, the saddle point $\left(k_{m r}, \omega_{V_{g}}\left(k_{m r}\right)\right)$ makes a 
contribution to the instability of the flow along the ray $x=x_{0}+V_{g} t$. This is the most unstable contribution among the contributions along all unstable rays. This follows from the fact that no $k$-root can cross the real $k$-axis, for $\omega$ with $\operatorname{Im} \omega>\operatorname{Im} \omega_{V_{g}}\left(k_{m r}\right)$. Therefore, $\operatorname{Im} \omega_{V_{g}}\left(k_{m r}\right)$ is the maximum growth rate.

The technique proceeds by following the movement of the saddle point of $\omega_{V}(k)$ in the complex $k$-plane starting with the point $\left(k_{m r}, 0\right)$ when the ray velocity $V$ varies continuously starting with $V_{g}$. Since $\omega_{V}(k)=\omega(k)-V k$, this can be done by computing the $\omega$-roots of $D(k, \omega)=0$ and following the movement of the point in the $k$-plane at which $\mathrm{d} \omega / \mathrm{d} k=V$ holds. At the outset, the unstable branch of $\omega$, for real $k$, is computed, the point $k_{m r}$ is found and $V_{g}$ is computed. Thus, the saddle point $k_{m r}$ on the real $k$-axis is the starting point in this procedure. The movement of the saddle point in the $k$-plane satisfying $\omega^{\prime}(k)=V$ is traced by varying the imaginary part of $k$ with small steps starting with $\operatorname{Im} k=0$, i.e. $\operatorname{Im} k=k_{i}^{1}, k_{i}^{2}, \ldots$, computing at each step $n$ the frequency $\omega^{n}\left(k_{r}\right) \equiv \omega\left(k_{r}+\mathrm{i} k_{i}^{n}\right)$, and finding a point at which Im $\omega^{n}\left(k_{r}\right)$ has a maximum connected by continuity with the maximum computed in the previous step. Here the superscript $n$ is a step number. Let $k_{m r}^{n}$ be the point of maximum at step $n$ which satisfies $\mathrm{d}\left[\operatorname{Im} \omega^{n}\left(k_{m r}\right)\right] / \mathrm{d} k_{r}=0$. Then at step $n$ the ray velocity is computed as $V^{n}=\mathrm{d}\left[\operatorname{Re} \omega^{n}\left(k_{m r}^{n}\right)\right] / \mathrm{d} k_{r}$. The growth rate along the ray $x=x_{0}+V^{n} t$ is $\omega_{i}\left(V^{n}\right)=\operatorname{Im} \omega^{n}\left(k_{m r}^{n}\right)-V^{n} k_{i}^{n}$, and the frequency of oscillations is $\omega_{r}\left(V^{n}\right)=-\left[\operatorname{Re} \omega^{n}\left(k_{m r}^{n}\right)-V^{n} k_{m r}^{n}\right]$.

Computations are performed for a sequence of increasing $k_{i}^{n}, n=1,2, \ldots$, starting with $k_{i}=0$, and similarly for a sequence of decreasing $k_{i}^{j}, j=1,2, \ldots$, until the points are reached for which the growth rate vanishes (Simmons \& Hoskins 1979; Deissler 1987; Brevdo 1995). Let these points correspond to the ray velocities $V_{l}$ and $V_{r}$, with $V_{l}<V_{g}<V_{r}$. It is then assumed that (i) the interval $\left(V_{l}, V_{r}\right)$ consists of unstable ray velocities, and (ii) for each ray velocity this procedure computes the maximum growth rate.

\section{REFERENCES}

Benjamin, T. B. 1957 Wave formation in laminar flow down an inclined plane. J. Fluid Mech. 2, $554-574$.

Benjamin, T. B. 1961 The development of three-dimensional disturbances in an unstable film of liquid flowing down an inclined plane. J. Fluid Mech. 10, 401-419.

Benney, D. J. 1966 Long waves on liquid films. J. Math. Phys. 45, 150-155.

BERs, A. 1973 Theory of absolute and convective instabilities. In International Congress on Waves and Instabilities in Plasma, Innsbruck, Austria (ed. G. Auer \& F. Cap), pp. B1-B52.

Brevdo, L. 1988 A study of absolute and convective instabilities with an application to the Eady model. Geophys. Astrophys. Fluid Dyn. 40, 1-92.

Brevdo, L. 1991 Three-dimensional absolute and convective instabilities, and spatially amplifying waves in parallel shear flows. Z. Angew. Math. Phys. 42, 911-942.

Brevdo, L. 1992a Spatially amplifying waves in plane Poiseuille flow. Z. Angew. Math. Mech. 72, $163-174$.

Brevdo, L. $1992 b$ A note on the Gaster transformation. Z. Angew. Math. Mech. 72, 305-306.

Brevdo, L. 1995 Convectively unstable wave packets in the Blasius boundary layer. Z. Angew. Math. Mech. 75, 423-436.

BREVDO, L. 1998 Wave packets, signaling and resonances in a homogeneous waveguide. J. Elasticity 49, 201-237.

Brevdo, L. \& Bridges, T. J. 1996 Absolute and convective instabilities of spatially periodic flows. Phil. Trans. R. Soc. Lond. A 354, 1027-1064.

Bridges, T. J. \& Morris, P. J. 1984 Differential eigenvalue problem in which the parameter appears nonlinearly. J. Comput. Phys. 55, 437-460. 
BRIGgS, R. J. 1964 Electron-Stream Interaction with Plasmas. MIT Press.

Chandrasekhar, S. 1961 Hydrodynamic and Hydromagnetic Stability. Clarendon.

Chang, H.-C. 1994 Wave evolution on a falling film. Ann. Rev. Fluid Mech. 26, 103-136.

Chang, H.-C. \& Demekhin, E. A. 1995 Repulsive dynamics of solitary pulses. Proc. IUTAM/ISIMM Symposium on Structure and Dynamics of Nonlinear Waves in Fluids, pp. 24-41. World Scientific.

Chang, H.-C. \& Demekhin, E. A. 1996 Solitary wave formation and dynamics on falling films. Adv. Appl. Mech. 32, 1-58.

Chang, H.-C., Demekhin, E. A. \& Kopelevich, D. I. 1993 Nonlinear evolution of waves on a vertically falling film. J. Fluid Mech. 250, 433-480.

Deissler, R. J. 1987 The convective nature of instability in plane Poiseuille flow. Phys. Fluids 30, 2303-2305.

Drazin, P. G. \& ReID, W. H. 1981 Hydrodynamic Stability. Cambridge University Press.

Floryan, J. M., Davis, S. H. \& Kelly, R. E. 1987 Instabilities of a liquid film flowing down a slightly inclined plane. Phys. Fluids 30, 983-989.

GASTER, M. 1962 A note on the relation between temporally-increasing and spatially-increasing disturbances in hydrodynamic stability. J. Fluid Mech. 14, 222-224.

Gaster, M. \& Grant, I. 1975 An experimental investigation of the formation and development of a wavepacket in a laminar boundary layer. Proc. R. Soc. Lond. A 347, 253-269.

HuERre, P. 1987 Spatio-temporal instabilities in closed and open flows. In Instabilities and Nonequilibrium Structures (ed. E. Tirapegui \& D. Villaroel), pp. 141-177. Reidel.

Huerre, P. \& Monkewitz, P. A. 1985 Absolute and convective instabilities in free shear layers. $J$. Fluid Mech. 159, 151-168.

Huerre, P. \& Monkewitz, P. A. 1990 Local and global instabilities in spatially developing flows. Ann. Rev. Fluid Mech. 22, 473-537.

Joo, S. W. \& DAvis, S. H. 1992 Instabilities of three-dimensional viscous falling films. J. Fluid Mech. 242, 529-547.

KapitZA, P. L. \& Kapitza, S. P. 1949 Wave flow of thin layers of a viscous fluid. Zh. Eksper. Teor. Fiz. 19, 105-120. Also in Collected Papers of P. L. Kapitza (ed. D. Ter Haar), pp. 690-709. Pergamon, 1965.

Kupfer, K. Bers, A. \& RAM, A. K. 1987 The cusp map in the complex-frequency plane for absolute instabilities. Phys. Fluids 30, 3075-3082.

LEE, J.-J. \& MeI, C. C. 1996 Stationary waves on an inclined sheet of viscous fluid at high Reynolds and moderate Weber numbers. J. Fluid Mech. 307, 191-229.

Lin, S. P. 1974 Finite amplitude side-band stability of a viscous film. J. Fluid Mech. 63, 417-429.

LingwoOD, R. J 1997 On the application of the Briggs and steepest-descent methods to a boundarylayer flow. Stud. Appl. Maths 98, 213-254.

LiU, J. \& Gollub, J. P. 1993 Onset of spatially chaotic waves on flowing films. Phys. Rev. Lett. 70, 2289-2292.

LiU, J. \& Gollub, J. P. 1994 Solitary wave dynamics of film flows. Phys. Fluids 6, 1702-1712.

LiU, J., Paul, J. D. \& Gollub, J. P. 1993 Measurements of the primary instabilities of film flows. $J$. Fluid Mech. 250, 69-101.

Liu, J., Schneider, J. B. \& Gollub, J. P. 1995 Three-dimensional instabilities in film flows. Phys. Fluids 7, 55-67.

MeI, C. C. 1966 Nonlinear gravity waves in a thin sheet of viscous fluid. J. Math. Phys. 45, 266-288.

Nusselt, W. 1916 Die Oberflächenkondensation des Wasserdampfes. Z. Ver. Deutsch. Ing. 27, 541546.

OrsZAG, S. 1971 Accurate solution of the Orr-Sommerfeld equation. J. Fluid Mech. 50, 689-703.

Pearlstein, A. J. \& Goussis, D. A. 1988 Efficient transformation of certain singular polynomial matrix eigenvalue problems. J. Comput. Phys. 78, 305-312.

Roskes, G. J. 1970 Three-dimensional long waves on a liquid film. Phys. Fluids 13, 1440-1445.

Simmons, A. J. \& Hoskins, B. J. 1979 The downstream and upstream development of unstable baroclinic waves J. Atmos. Sci. 36, 1239-1254.

Stokes, G. G. 1847 On the theory of oscillatory waves. Trans. Camb. Phil. Soc. 8, 441-455.

YIH, C. S. 1955 Stability of parallel laminar flow with a free surface. In Proc. 2nd US Congr. on Applied Mechanics, pp. 623-628. ASME. 